\title{
MID-INFRARED SPECTRAL DIAGNOSTICS OF NUCLEAR AND EXTRANUCLEAR REGIONS IN NEARBY GALAXIES
}

\author{
D. A. Dale, ${ }^{1}$ J. D. T. Smith, ${ }^{2}$ L. Armus,${ }^{3}$ B. A. Buckalew,${ }^{3}$ G. Helou, ${ }^{3}$ R. C. Kennicutt JR., ${ }^{2,4}$ J. Moustakas, ${ }^{2}$ \\ H. Roussel, ${ }^{3,5}$ K. Sheth, ${ }^{3}$ G. J. Bendo, ${ }^{2,6}$ D. Calzetti, ${ }^{7}$ B. T. Draine, ${ }^{8}$ C. W. Engelbracht, ${ }^{2}$ \\ K. D. Gordon, ${ }^{2}$ D. J. Hollenbach, ${ }^{9}$ T. H. Jarrett ${ }^{3}$ L. J. Kewley, ${ }^{10}$ C. Leitherer, ${ }^{7}$ \\ A. Li, ${ }^{11}$ S. Malhotra, ${ }^{12}$ E. J. Murphy, ${ }^{13}$ and F. Walter ${ }^{5}$ \\ Received 2006 February 24; accepted 2006 March 31
}

\begin{abstract}
Mid-infrared diagnostics are presented for a large portion of the Spitzer Infrared Nearby Galaxies Survey (SINGS) sample plus archival data from ISO and Spitzer. The SINGS data set includes low- and high-resolution spectral maps and broadband imaging in the infrared for over 160 nuclear and extranuclear regions within 75 nearby galaxies spanning a wide range of morphologies, metallicities, luminosities, and star formation rates. Our main result is that these mid-infrared diagnostics effectively constrain a target's dominant power source. The combination of a highionization line index and PAH strength serves as an efficient discriminant between AGNs and star-forming nuclei, confirming progress made with $I S O$ spectroscopy on starbursting and ultraluminous infrared galaxies. The sensitivity of Spitzer allows us to probe fainter nuclear and star-forming regions within galaxy disks. We find that both starforming nuclei and extranuclear regions stand apart from nuclei that are powered by Seyfert or LINER activity. In fact, we identify areas within four diagnostic diagrams containing $>90 \%$ Seyfert/LINER nuclei or $>90 \% \mathrm{H}$ II regions/ $\mathrm{H}$ II nuclei. We also find that, compared to starbursting nuclei, extranuclear regions typically separate even further from AGNs, especially for low-metallicity extranuclear environments. In addition, instead of the traditional midinfrared approach to differentiating between AGNs and star-forming sources that utilizes relatively weak highionization lines, we show that strong low-ionization cooling lines of X-ray-dominated regions like [Si II] $34.82 \mu \mathrm{m}$ can alternatively be used as excellent discriminants. Finally, the typical target in this sample shows relatively modest interstellar electron density $\left(\sim 400 \mathrm{~cm}^{-3}\right)$ and obscuration $\left(A_{V} \sim 1.0 \mathrm{mag}\right.$ for a foreground screen), consistent with a lack of dense clumps of highly obscured gas and dust residing in the emitting regions.
\end{abstract}

Subject headings: galaxies: active — galaxies: nuclei — H II regions — infrared: galaxies — infrared: ISM

Online material: color figures, machine-readable table

\section{INTRODUCTION}

The goal of this study is to explore whether mid-infrared diagnostics developed for luminous/ultraluminous infrared galaxies (LIRGs/ULIRGs) and bright Galactic $\mathrm{H}$ II regions can be improved on and extended to the nuclear and extranuclear regions within normal and infrared-faint galaxies. A traditional method for characterizing a galaxy's nuclear power source uses ratios of optical emission lines such as [O II] $\lambda 3727, \mathrm{H} \beta \lambda 4861$,

\footnotetext{
1 Department of Physics and Astronomy, University of Wyoming, Laramie, WY 82071; ddale@uwyo.edu.

2 Steward Observatory, University of Arizona, 933 North Cherry Avenue, Tucson, AZ 85721.

3 California Institute of Technology, MC 314-6, Pasadena, CA 91101.

4 Institute of Astronomy, University of Cambridge, Madingley Road, Cambridge CB3 OHA, UK.

${ }_{5}^{5}$ Max-Planck-Institut für Astronomie, Königstuhl 17, 69117 Heidelberg, Germany.

${ }^{6}$ Astrophysics Group, Imperial College, Blackett Laboratory, Prince Consort Road, London SW7 2AZ, UK.

7 Space Telescope Science Institute, 3700 San Martin Drive, Baltimore, MD 21218.

8 Princeton University Observatory, Peyton Hall, Princeton, NJ 08544.

9 NASA Ames Research Center, MS 245-6, Moffett Field, CA 94035.

10 Institute for Astronomy, University of Hawaii, 2680 Woodlawn Drive, Honolulu, HI 96822.

11 Department of Physics and Astronomy, University of Missouri, Columbia, MO 65211.

12 Department of Physics and Astronomy, Arizona State University, Box 871504, Tempe, AZ 85287.

13 Department of Astronomy, Yale University, P.O. Box 208101, New Haven, CT 06520.
}

[O III] $\lambda 5007$, [O I] $\lambda 6300, \mathrm{H} \alpha \lambda 6563,[\mathrm{~N}$ II] $\lambda 6584$, and [S II] $\lambda \lambda 6716,6731$ (e.g., Baldwin et al. 1981; Veilleux \& Osterbrock 1987; Ho et al. 1997b; Kewley et al. 2001; Kauffmann et al. 2003). A plot of [O III] $/ \mathrm{H} \beta$ versus [ $\mathrm{N}$ II] $/ \mathrm{H} \alpha$, for example, will typically separate Seyfert galaxies, LINERs, and starburst nuclei. Since nuclei are often heavily enshrouded by dust, especially in LIRGs and ULIRGs, an important limitation to galaxy optical diagnostics is the effect of extinction. In anticipation of the data stream from space-based infrared platforms, early theoretical work with photoionization models showed that infrared ionic fine-structure line ratios could profitably enable astronomers to approach galaxy classification from a new perspective (e.g., Voit 1992; Spinoglio \& Malkan 1992). The advent of sensitive infrared line data from the Infrared Space Observatory (ISO) was an important first step to peering more deeply into buried nuclear sources (Genzel et al. 1998; Laurent et al. 2000; Sturm et al. 2002; Peeters et al. 2004b). Genzel and collaborators were the first to show that ionization-sensitive indices based on mid-infrared line ratios correlate with the strength of polycyclic aromatic hydrocarbon (PAH) emission features. Active galactic nuclei (AGNs) in particular show weak PAH and large ratios of high- to low-ionization line emission. Interestingly, while Genzel et al. (1998) found that $70 \%-80 \%$ of their ULIRG sample is mainly powered by starburst activity, the percentage appears to drop for higher luminosity ULIRGs (e.g., Veilleux et al. 1999; but see also Farrah et al. 2003). Taniguchi et al. (1999) and Lutz et al. (1999) suggest that optical and infrared classifications agree if the nuclei of LINER-like ULIRGs are in fact dominated by shocks driven by powerful supernova winds. Mid-infrared 
lines observed by ISO were also used to probe the physical characteristics and evolution of purely starbursting nuclei (Thornley et al. 2000; Verma et al. 2003) and Galactic H II regions (Vermeij $\&$ van der Hulst 2002; Giveon et al. 2002). An important result stemming from these efforts is that stellar aging effects appear to result in $\mathrm{H}$ II regions generally having higher excitations than starbursting nuclei.

The unprecedented sensitivity and angular resolution afforded by the Spitzer Space Telescope allow an even more detailed view into the nature of galactic nuclei (e.g., Armus et al. 2004; Smith et al. 2004). The Spitzer Infrared Nearby Galaxies Survey (SINGS) takes full advantage of Spitzer's capabilities by executing a comprehensive, multiwavelength survey of 75 nearby galaxies spanning a wide range of morphologies, metallicities, luminosities, and star formation activity levels (Kennicutt et al. 2003). The sensitivity of Spitzer, coupled with the proximity of the SINGS sample, allows dwarf galaxy systems fainter than $L_{\mathrm{FIR}} \sim 10^{7} L_{\odot}$ to be spectroscopically probed in the infrared for the first time. In addition, prior to Spitzer the only individual extragalactic H II regions that were detectable with infrared spectroscopy resided in the Local Group (e.g., Giveon et al. 2002; Vermeij et al. 2002). In contrast, SINGS provides infrared spectroscopic data for nearly 100 extragalactic $\mathrm{H}$ II regions, residing in systems as near as Local Group members to galaxies as far as $\sim 25 \mathrm{Mpc}$. The SINGS data set thus samples a wider range of environments than previously observed with infrared spectroscopy. This diversity in the SINGS sample provides a huge range for exploring physical parameters with mid-infrared spectral diagnostics. The high-ionization lines historically used in such diagnostics, such as [O IV] $25.89 \mu \mathrm{m}$ and [ $\mathrm{Nev}] 14.32 \mu \mathrm{m}$, are relatively weak and can be difficult to detect in lower luminosity systems. Fortunately, high-ionization lines are not the only route to determining whether a galaxy harbors a strong AGN. Similar to how the [O I] $\lambda 6300$ and [O I] $63 \mu \mathrm{m}$ lines are AGN diagnostics (e.g., Dale et al. 2004a), we show below the utility of using the comparatively bright [Si II] $34.82 \mu \mathrm{m}$ midinfrared line as another effective tool for deciphering a galaxy's power source.

\section{THE SAMPLE}

\subsection{Galactic Nuclei}

The sample of nuclear targets analyzed in this study derives from the SINGS Third Data Release. These 50 nuclei come from a wide range of environments and galaxies: low-metallicity dwarfs; quiescent elliptical galaxies; dusty grand-design spiral galaxies; Seyfert galaxies, LINERs, and starbursting nuclei of normal galaxies; and systems within the Local Group and M81 group (see Table 1 and Kennicutt et al. 2003).

\subsection{Extranuclear Regions}

The 26 extranuclear sources studied in this work also come from the SINGS Third Data Release (see Table 2). These targets stem from the original set of 39 optically selected sources listed by Kennicutt et al. (2003). The optically selected OB/H II regions cover a large range of metallicity $\left(0.1-3 Z_{\odot}\right)$, extinctioncorrected ionizing luminosity $\left(10^{49}-10^{52}\right.$ photons $\left.\mathrm{s}^{-1}\right)$, extinction $\left(A_{V} \lesssim 4 \mathrm{mag}\right.$ ), radiation field intensity (ionization parameter $\log U=-2$ to -4 ; Habing 1968), ionizing stellar temperature $\left(T_{\text {eff }}=35-55 \mathrm{kK}\right)$, and local $\mathrm{H}_{2} / \mathrm{H}$ I ratio as inferred from $\mathrm{CO}$ $(<0.1$ to $>10)$. Additional extranuclear targets for the SINGS project have since been identified, based on their infrared properties, but their observations necessarily came later than the observations of the optically selected targets. Those "second look" data will be the focus of a future paper.

\section{THE DATA}

The full SINGS observing program and the data processing are described by Kennicutt et al. (2003), Smith et al. (2004), and Dale et al. (2005). Here we briefly summarize the spectral observations and data processing relevant to this paper.

\subsection{Spitzer Infrared Spectroscopy Observations and Data Processing}

High-resolution spectroscopy $(R \sim 600)$ was obtained in the Short-High $(10-19 \mu \mathrm{m})$ and Long-High $(19-37 \mu \mathrm{m})$ modules, and low-resolution spectroscopy $(R \sim 50-100)$ was obtained in the Short-Low $(5-14 \mu \mathrm{m})$ and Long-Low $(14-38 \mu \mathrm{m})$ modules (Houck et al. 2004a). Figure 1 shows example spectra for a variety of sources (Long-Low data are not used elsewhere in this work). Nuclei were generally mapped with a $3 \times 5$ grid (ShortHigh and Long-High) and a $1 \times 18$ grid (Short-Low), utilizing half-slit width and half-slit length steps. Extranuclear targets were observed with a similar scheme but with a $1 \times 9$ Short-Low grid. For a subset of nine sources with extended circumnuclear star formation we obtained slightly larger $(6 \times 10)$ Short-High nuclear maps. Owing to the different angular sizes subtended by the instruments, the resulting maps are approximately $57^{\prime \prime} \times 31^{\prime \prime}$ and $57^{\prime \prime} \times 18^{\prime \prime}$ in Short-Low (nuclear and extranuclear, respectively), $45^{\prime \prime} \times 33^{\prime \prime}$ in Long-High, and $23^{\prime \prime} \times 15^{\prime \prime}$ in Short-High (the 10 expanded Short-High nuclear maps are $40^{\prime \prime} \times 28^{\prime \prime}$ ). All integrations are $60 \mathrm{~s}$ per pointing, except the Short-Low nuclear maps, which are $14 \mathrm{~s}$ per pointing. The effective integrations are longer since each location was covered 2-4 times.

The individual data files for a given spectral map were assembled into spectral cubes using the software CUBISM (Kennicutt et al. 2003; J. D. T. Smith et al. 2006, in preparation). The cube input data were preprocessed using version S12.0 of the Spitzer Science Center pipeline. Various postprocessing steps within CUBISM are described by Smith et al. (2004). Short-Low sky subtraction was enabled via the extended off-source wings of the Short-Low module (and occasionally using spatially and temporally proximate data from the archive when our off-source wings do not extend to the sky). Several cross-checks on the flux calibration were made between Short-Low, Short-High, Long-Low, Long-High, MIPS, and IRAC data. The absolute flux calibration uncertainty for the spectral data is estimated to be $25 \%$; the uncertainty in line flux ratios is $\sim 10 \%$.

Although the Short-High, Long-High, and Short-Low cubes all span different solid angles, the same matched extraction apertures were used for all cubes: one-dimensional spectra were extracted from the three-dimensional data using $\sim 23^{\prime \prime} \times 15^{\prime \prime}$ apertures. Furthermore, the extraction apertures are centered on the optically derived coordinates listed by Kennicutt et al. (2003); the optical coordinates generally coincide with the infrared emission peak.

Emission-line and PAH feature fluxes and equivalent widths are derived from continuum-subtracted Gaussian fits to the lines and first- or second-order polynomial fits to the continua.

\subsection{Archival Spectroscopy}

ISO SWS and Spitzer IRS line fluxes are drawn from the literature for a wide variety of sources including Galactic, Magellanic Cloud, and Local Group H II regions ( Vermeij et al. 2002; Giveon et al. 2002; Peeters et al. 2002) and starburst and active galaxies (Genzel et al. 1998; Sturm et al. 2002; Verma et al. 2003; Armus et al. 2004; Peeters et al. 2004b; Weedman et al. 2005; Haas et al. 2005). Equivalent widths of the $6.2 \mu \mathrm{m}$ PAH feature were extracted from archival ISO PHOT and Spitzer IRS data, when 
TABLE 1

Nuclear Emission Line Flux Intensities and $6.2 \mu \mathrm{m}$ PAH Feature Equivalent Widths

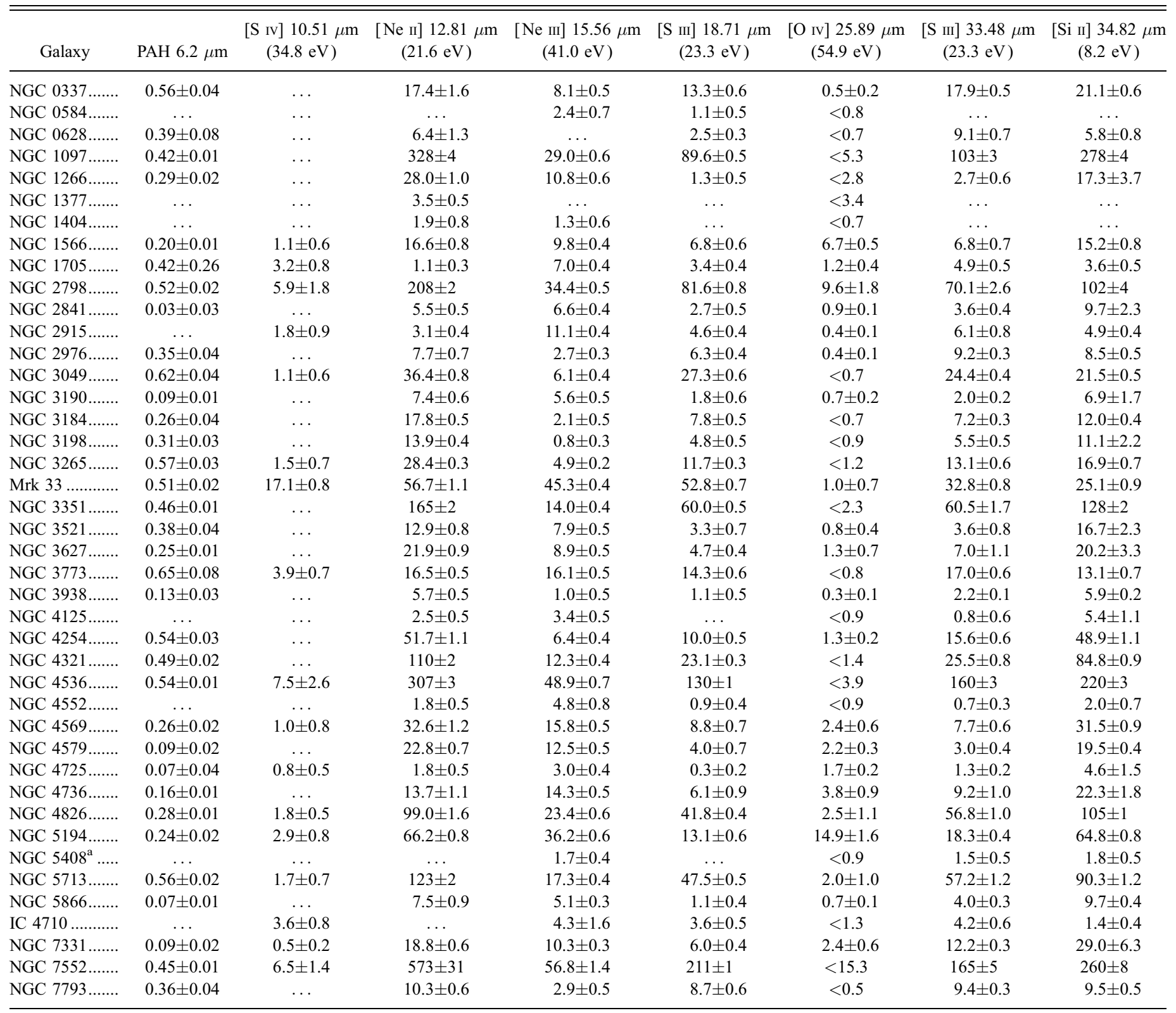

Notes.-Fluxes and their (statistical) uncertainties are averaged over $\sim 23^{\prime \prime} \times 15^{\prime \prime}$ and listed in units of $10^{-9} \mathrm{~W} \mathrm{~m}^{-2} \mathrm{sr}^{-1}$. Calibration uncertainties are an additional $\sim 30 \%$. The $6.2 \mu \mathrm{m}$ PAH feature equivalent width is given in units of microns. Eight galaxies from the SINGS Third Data Release are not listed in this table. No Short-Low, Short-High, or Long-High data were taken for the optical centers of Holmberg II, IC 2574, DDO 154, and NGC 6822. DDO 053, Holmberg IX, DDO 165, and NGC 5398 (Tololo 89) were nondetections. The $3 \sigma$ upper limits are provided for nondetections of [O IV] $\sim 25.89 \mu \mathrm{m}$.

${ }^{a}$ The infrared emission peaks outside of the field of view of the spectral maps.

available. Note that, for a given source, only data from Spitzer or only data from ISO are used; cross observatory data are not used in this analysis. The fields of view of the ISO PHOT and ISO SWS apertures $\left(24^{\prime \prime} \times 24^{\prime \prime}\right.$ and $14^{\prime \prime} \times 20^{\prime \prime}$ to $20^{\prime \prime} \times 30^{\prime \prime}$, respectively) provide a reasonable match to the $\sim 23^{\prime \prime} \times 15^{\prime \prime}$ extraction apertures described in $\S 3.1$. Table 3 provides the list of archival sources used in this work.

\subsection{Spitzer Broadband Imaging: $24 \mu \mathrm{m}$}

The SINGS project did not obtain nearby sky observations in HighRes mode, so if the underlying hot dust continuum emission is detected, the foreground/background continuum is not subtracted. SINGS IRS HighRes spectroscopy is designed to measure line fluxes; thus, line equivalent width measures via
HighRes are biased by the unsubtracted sky emission. On the other hand, the SINGS program includes extensive MIPS (Rieke et al. 2004) $24 \mu \mathrm{m}$ broadband imaging of all galaxies; the $24 \mu \mathrm{m}$ data can be used to normalize line fluxes. Sky-subtracted MIPS $24 \mu \mathrm{m}$ fluxes are extracted from aperture cutouts matched to the Short-High field of view ( $\S 3.1$ ). See Kennicutt et al. (2003) and Dale et al. (2005) for further details of the SINGS broadband imaging.

\subsection{Optical Spectroscopy Observations and Data Processing}

Optical spectrophotometry has been obtained for the SINGS project at the Steward Observatory Bok $2.3 \mathrm{~m}$ and the Cerro Tololo Inter-American Observatory (CTIO) $1.5 \mathrm{~m}$ telescopes (Moustakas \& Kennicutt 2006). A suite of spectral drift scans, 
TABLE 2

Extranuclear Emission Line Flux Intensities and $6.2 \mu \mathrm{m}$ PAH Feature Equivalent Widths

\begin{tabular}{|c|c|c|c|c|c|c|c|c|}
\hline Galaxy & PAH $6.2 \mu \mathrm{m}$ & $\begin{array}{c}{[\mathrm{S} \text { Iv }] 10.51 \mu \mathrm{m}} \\
\quad(34.8 \mathrm{eV})\end{array}$ & $\begin{array}{c}{[\mathrm{Ne} \mathrm{II}] 12.81 \mu \mathrm{m}} \\
\quad(21.6 \mathrm{eV})\end{array}$ & $\begin{array}{c}{[\mathrm{Ne} \text { III] } 15.56 \mu \mathrm{m}} \\
(41.0 \mathrm{eV})\end{array}$ & $\begin{array}{c}{[\mathrm{S} \text { III] } 18.71 \mu \mathrm{m}} \\
(23.3 \mathrm{eV})\end{array}$ & $\begin{array}{c}{[\mathrm{O} \text { Iv }] 25.89 \mu \mathrm{m}} \\
(54.9 \mathrm{eV})\end{array}$ & $\begin{array}{c}\text { [S III] } 33.48 \mu \mathrm{m} \\
\quad(23.3 \mathrm{eV})\end{array}$ & $\begin{array}{c}{\left[\begin{array}{l}\mathrm{Si} \\
\mathrm{II}]\end{array} 34.82 \mu \mathrm{m}\right.} \\
(8.2 \mathrm{eV})\end{array}$ \\
\hline NGC 5194 CCM $107 \ldots \ldots \ldots \ldots \ldots . .$. & $0.47 \pm 0.02$ & & $32.0 \pm 0.6$ & $2.3 \pm 0.6$ & $10.6 \pm 0.5$ & $1.7 \pm 0.3$ & $18.3 \pm 0.5$ & $34.1 \pm 0.5$ \\
\hline NGC 5194 CCM $072 \ldots \ldots \ldots \ldots \ldots . .$. & $0.61 \pm 0.02$ & $1.7 \pm 0.5$ & $71.9 \pm 1.0$ & $3.8 \pm 0.4$ & $29.4 \pm 0.6$ & $1.4 \pm 0.8$ & $36.2 \pm 0.5$ & $49.2 \pm 0.7$ \\
\hline NGC 5194 CCM 071 ............... & $0.66 \pm 0.02$ & $\ldots$ & $44.3 \pm 0.7$ & $5.6 \pm 0.5$ & $16.6 \pm 0.7$ & $1.3 \pm 0.4$ & $25.0 \pm 0.6$ & $41.2 \pm 0.5$ \\
\hline NGC 5194 CCM $001 \ldots \ldots \ldots \ldots . .$. & $0.69 \pm 0.02$ & $\ldots$ & $16.4 \pm 1.1$ & $3.3 \pm 0.5$ & $8.2 \pm 0.3$ & $0.6 \pm 0.2$ & $13.7 \pm 0.5$ & $21.7 \pm 0.9$ \\
\hline NGC 5194 CCM $010 \ldots \ldots \ldots \ldots . .$. & $0.69 \pm 0.03$ & $\ldots$ & $39.2 \pm 0.7$ & $6.1 \pm 0.4$ & $19.8 \pm 0.5$ & $0.5 \pm 0.3$ & $29.0 \pm 0.5$ & $37.7 \pm 0.7$ \\
\hline NGC 5194 CCM 071A ........... & $0.52 \pm 0.02$ & $\ldots$ & $21.3 \pm 0.6$ & $5.7 \pm 0.4$ & $11.0 \pm 0.5$ & $<0.6$ & $14.4 \pm 2.1$ & $12.6 \pm 0.4$ \\
\hline 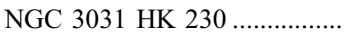 & $0.44 \pm 0.03$ & . & $4.1 \pm 0.5$ & $0.7 \pm 0.5$ & $2.7 \pm 0.4$ & $<0.7$ & $3.6 \pm 0.5$ & $4.0 \pm 0.4$ \\
\hline NGC 3031 HK $343 \ldots \ldots \ldots \ldots \ldots . . . .$. & $0.38 \pm 0.03$ & $\ldots$ & $7.5 \pm 3.1$ & $3.6 \pm 0.4$ & $7.0 \pm 0.9$ & $<0.8$ & $9.8 \pm 0.8$ & $7.0 \pm 0.6$ \\
\hline NGC 3031 HK $453 \ldots \ldots \ldots \ldots \ldots . . .$. & $0.65 \pm 0.05$ & $\ldots$ & $9.0 \pm 0.6$ & $3.4 \pm 0.4$ & $8.8 \pm 0.9$ & $<0.8$ & $11.7 \pm 2.0$ & $9.3 \pm 0.6$ \\
\hline NGC 3031 HK $268 \ldots \ldots \ldots \ldots \ldots \ldots . . .$. & $0.55 \pm 0.03$ & $1.2 \pm 0.7$ & $15.1 \pm 0.8$ & $6.0 \pm 0.4$ & $13.4 \pm 0.7$ & $<0.8$ & $17.3 \pm 0.7$ & $13.0 \pm 0.6$ \\
\hline NGC 3031 HK $652 \ldots \ldots \ldots \ldots \ldots . .$. & $0.68 \pm 0.06$ & $\ldots$ & $10.3 \pm 0.7$ & $2.7 \pm 0.5$ & $9.2 \pm 1.1$ & $<0.8$ & $10.7 \pm 0.8$ & $11.8 \pm 0.6$ \\
\hline NGC 3031 HK 741 ..................... & $0.75 \pm 0.05$ & $\ldots$ & $8.9 \pm 0.8$ & $1.6 \pm 0.4$ & $7.3 \pm 0.4$ & $<0.9$ & $7.6 \pm 0.7$ & $6.5 \pm 0.7$ \\
\hline NGC 3031 Munch 1 ................... & $2.98 \pm 2.29$ & $\ldots$ & $\ldots$ & $1.4 \pm 0.2$ & $\ldots$ & $<1.0$ & $\ldots$ & $\ldots$ \\
\hline 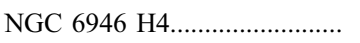 & $0.65 \pm 0.02$ & $3.6 \pm 0.7$ & $16.6 \pm 0.6$ & $17.5 \pm 0.5$ & $14.7 \pm 0.4$ & $1.1 \pm 0.2$ & $14.2 \pm 0.6$ & $14.6 \pm 0.5$ \\
\hline NGC 6946 HK $3 \ldots \ldots \ldots \ldots \ldots \ldots \ldots . .$. & $0.63 \pm 0.03$ & $8.8 \pm 1.3$ & $28.2 \pm 0.4$ & $31.9 \pm 0.4$ & $30.6 \pm 0.4$ & $0.9 \pm 0.2$ & $39.0 \pm 0.5$ & $25.1 \pm 0.5$ \\
\hline 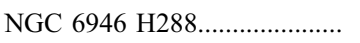 & $0.81 \pm 0.04$ & $\ldots$ & $18.1 \pm 0.4$ & $8.5 \pm 0.4$ & $14.0 \pm 0.5$ & $<0.6$ & $19.3 \pm 0.3$ & $11.8 \pm 0.2$ \\
\hline NGC 6946 H40............................. & $0.84 \pm 0.03$ & $1.9 \pm 0.8$ & $18.8 \pm 0.4$ & $8.5 \pm 0.4$ & $15.0 \pm 0.4$ & $0.2 \pm 0.1$ & $17.3 \pm 0.3$ & $15.2 \pm 0.5$ \\
\hline 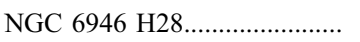 & $1.05 \pm 0.45$ & $\ldots$ & $9.4 \pm 0.5$ & $2.9 \pm 0.3$ & $6.8 \pm 0.5$ & $<0.5$ & $9.0 \pm 0.3$ & $8.2 \pm 0.4$ \\
\hline 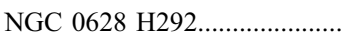 & $0.55 \pm 0.02$ & $2.1 \pm 0.9$ & $25.6 \pm 0.7$ & $2.7 \pm 0.4$ & $20.1 \pm 0.6$ & $<1.0$ & $27.4 \pm 0.5$ & $13.1 \pm 0.7$ \\
\hline 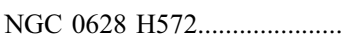 & $0.69 \pm 0.03$ & $\ldots$ & $12.5 \pm 0.7$ & $2.6 \pm 0.5$ & $8.1 \pm 0.4$ & $<0.9$ & $16.3 \pm 0.5$ & $8.2 \pm 0.6$ \\
\hline NGC 0628 H627........................... & $0.60 \pm 0.03$ & $3.8 \pm 1.3$ & $11.5 \pm 0.7$ & $9.5 \pm 0.5$ & $10.6 \pm 0.4$ & $<0.9$ & $17.8 \pm 0.9$ & $9.6 \pm 0.5$ \\
\hline 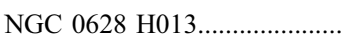 & $0.52 \pm 0.07$ & $\ldots$ & $4.1 \pm 0.7$ & $2.2 \pm 0.3$ & $4.9 \pm 0.4$ & $<0.7$ & $11.3 \pm 1.3$ & $2.9 \pm 0.4$ \\
\hline Holmberg II HSK $45 \ldots \ldots \ldots \ldots \ldots . . .$. & $\ldots$ & $\ldots$ & $\ldots$ & $3.4 \pm 0.5$ & $3.2 \pm 0.4$ & $<0.9$ & $2.9 \pm 0.7$ & $2.8 \pm 0.5$ \\
\hline Holmberg II HSK $67 . \ldots \ldots \ldots \ldots \ldots . . . .$. & $\ldots$ & $\ldots$ & $\ldots$ & $0.7 \pm 0.2$ & $\ldots$ & $<0.7$ & $0.6 \pm 0.2$ & $0.9 \pm 0.3$ \\
\hline Holmberg II HSK $70 \ldots \ldots \ldots \ldots \ldots \ldots . .$. & $\ldots$ & $\ldots$ & $\ldots$ & $1.1 \pm 0.5$ & $0.6 \pm 0.2$ & $<0.9$ & $0.4 \pm 0.2$ & $1.7 \pm 0.5$ \\
\hline Holmberg II HSK $07 \ldots \ldots \ldots \ldots \ldots . . .$. & $\ldots$ & & $\ldots$ & $1.1 \pm 0.6$ & $0.8 \pm 0.5$ & $<1.0$ & $\ldots$ & $\ldots$ \\
\hline
\end{tabular}

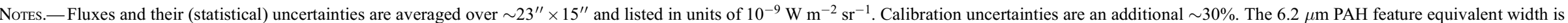
given in units of microns. The $3 \sigma$ upper limits are provided for nondetections of [O $\mathrm{IV}] \sim 25.89 \mu \mathrm{m}$. 


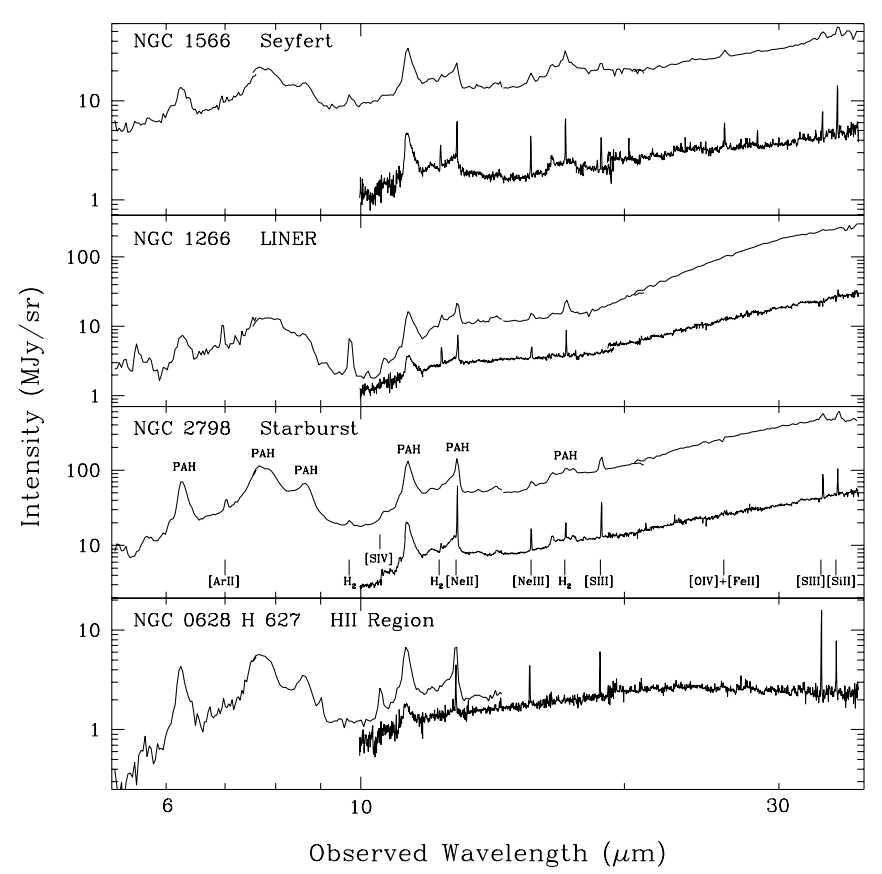

Fig. 1.-Examples of low- and high-resolution spectra for four different types of environments found within the SINGS sample. The IRS modules span wavelengths $\sim 5-7 \mu \mathrm{m}$ (Short-Low2), 7-14 $\mu \mathrm{m}$ (Short-Low1), $\sim 14-21 \mu \mathrm{m}$ (Long-Low2), $\sim 21-40 \mu \mathrm{m}$ (Long-Low1), $\sim 10-19 \mu \mathrm{m}$ (Short-High), and $\sim 19-37 \mu \mathrm{m}$ (Long-High). Each spectral segment has been extracted from a $\sim 23^{\prime \prime} \times 15^{\prime \prime}$ region. The high-resolution data are scaled downward by $1.0 \mathrm{dex}$ for clarity. The variable offset between the low- and high-resolution data is largely due to the lack of sky subtraction for the high-resolution data. [See the electronic edition of the Journal for a color version of this figure.]

centered on the nuclei, were taken to spatially map the various regions covered with the IRS spectroscopy program (for more details see Kennicutt et al. 2003). The optical spectroscopy has spectral resolution of $\sim 8 \AA$ and covers $3600-7000 \AA$ so that the primary nebular emission lines can be studied (e.g., [O II]

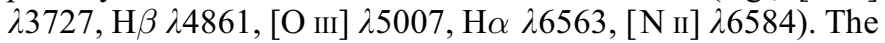
SINGS optical spectroscopy and emission-line measurements (fluxes, equivalent widths, metallicities, etc.) will be presented by J. Moustakas et al. (2006, in preparation).

This work utilizes optical spectral drift scans integrated over the central $20^{\prime \prime} \times 20^{\prime \prime}$ regions, approximately matching the $\sim 23^{\prime \prime} \times 15^{\prime \prime}$ circumnuclear regions over which the infrared line and PAH feature fluxes are extracted (see $\S 3.1)$.

\section{MEASURED QUANTITIES}

\subsection{High-Resolution Infrared Spectroscopy: Emission Lines}

Several forbidden lines are quite prominent in many of the SINGS nuclear and extranuclear high-resolution spectra ([ $\mathrm{Ne}$ II] $12.81 \mu \mathrm{m},[\mathrm{Ne}$ III] $15.56 \mu \mathrm{m}$, [S III] $18.71 \mu \mathrm{m}$, [S III] $33.48 \mu \mathrm{m}$, and [Si II] $34.82 \mu \mathrm{m}$ ) along with a few other higher ionization lines that are occasionally observed ([S Iv $] 10.51 \mu \mathrm{m},[\mathrm{Ne} \mathrm{v}]$ $14.32 \mu \mathrm{m}$, and [O Iv] $25.89 \mu \mathrm{m}$; Fig. 1).

All of these lines except [ $\mathrm{Si}$ II] $34.82 \mu \mathrm{m}$ are nebular lines from hydrogen gas ionized regions; [Si II] $34.82 \mu \mathrm{m}$ comes from a wider variety of regions, including both ionized gas and warm atomic gas, such as photodissociation regions and X-ray-dominated regions (e.g., Hollenbach \& Tielens 1999). A series of interstellar molecular hydrogen lines are also detected in the SINGS spectra; these are explored in a separate paper (H. Roussel et al. 2006, in preparation). The line fluxes utilized in this work are listed in Tables 1 and 2. Eight galaxies from the SINGS Third Data Re-
TABLE 3

Archival Sources

\begin{tabular}{|c|c|c|}
\hline Object & Type & References \\
\hline 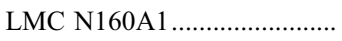 & LMC H II & Vermeij et al. (2002) \\
\hline 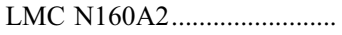 & LMC H II & Vermeij et al. (2002) \\
\hline 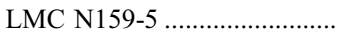 & LMC H II & Vermeij et al. (2002) \\
\hline 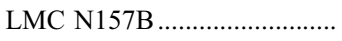 & LMC H II & Vermeij et al. (2002) \\
\hline 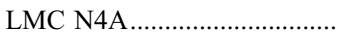 & LMC H II & Vermeij et al. (2002) \\
\hline LMC N11A ................. & LMC H II & Vermeij et al. (2002) \\
\hline LMC N83B ......... & LMC H II & Vermeij et al. (2002) \\
\hline 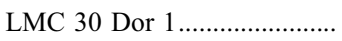 & LMC H II & Vermeij et al. (2002) \\
\hline LMC 30 Dor $2 \ldots \ldots$ & LMC H II & Vermeij et al. (2002) \\
\hline LMC 30 Dor $3 \ldots \ldots \ldots \ldots \ldots$ & LMC H II & Vermeij et al. (2002) \\
\hline LMC 30 Dor 4 & LMC H II & Vermeij et al. (2002) \\
\hline 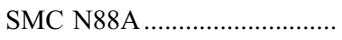 & SMC H II & Vermeij et al. (2002) \\
\hline 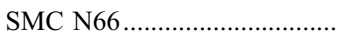 & SMC H II & Vermeij et al. (2002) \\
\hline 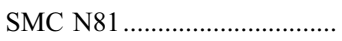 & SMC H II & Vermeij et al. (2002) \\
\hline NGC 0253 & $\mathrm{H}$ iI nucleus & Verma et al. (2003) \\
\hline IC 342 & $\mathrm{H}$ II nucleus & Verma et al. (2003) \\
\hline 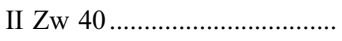 & $\mathrm{H}$ II nucleus & Verma et al. (2003) \\
\hline NGC $3034 \ldots \ldots \ldots \ldots \ldots \ldots \ldots$ & $\mathrm{H}$ II nucleus & Verma et al. (2003) \\
\hline 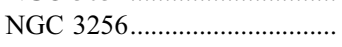 & $\mathrm{H}$ II nucleus & Verma et al. (2003) \\
\hline NGC 3690A & $\mathrm{H}$ II nucleus & Verma et al. (2003) \\
\hline NGC 3690B & $\mathrm{H}$ II nucleus & Verma et al. (2003) \\
\hline 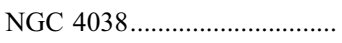 & $\mathrm{H}$ II nucleus & Verma et al. (2003) \\
\hline NGC $4945 \ldots \ldots \ldots \ldots \ldots \ldots \ldots$ & $\mathrm{H}$ II/Seyfert & Verma et al. (2003) \\
\hline NGC 5236 & $\mathrm{H}$ iI nucleus & Verma et al. (2003) \\
\hline NGC 5253 & $\mathrm{H}$ II nucleus & Verma et al. (2003) \\
\hline NGC 7552 & LINER/H II & Verma et al. (2003) \\
\hline 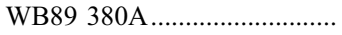 & Galactic H II & Giveon et al. (2002) \\
\hline 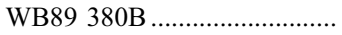 & Galactic H II & Giveon et al. (2002) \\
\hline WB89 399 & Galactic H II & Giveon et al. (2002) \\
\hline
\end{tabular}

Notes.-Table 3 is published in its entirety in the electronic edition of the Astrophysical Journal. A portion is shown here for guidance regarding its form and content.

lease are not listed in Table 1 because their nuclear regions were too faint to be observed (or detected if observed).

\subsection{Low-Resolution Infrared Spectroscopy: The $6.2 \mu \mathrm{m}$ PAH Feature}

The strength of a PAH feature depends on a complex combination of several parameters of the interstellar medium, some of which are interlinked: metallicity, dust column density, the distribution of sizes and ionization states in the dust grain population, and the intensity and hardness of the interstellar radiation field (e.g., Cesarsky et al. 1996; Thuan et al. 1999; Sturm et al. 2000; Li \& Draine 2001; Draine \& Li 2001; Houck et al. 2004b; Galliano et al. 2005; Engelbracht et al. 2005; Madden et al. 2006; Wu et al. 2006). Due to this sensitivity to the properties of the interstellar medium, PAH features in the mid-infrared have been used to characterize the physical state of a system. Previous efforts have utilized the $6.2 \mu \mathrm{m}$ feature (Laurent et al. 2000; Peeters et al. 2004b; Weedman et al. 2005), the $7.7 \mu \mathrm{m}$ feature (Genzel et al. 1998), or a combination of several PAH features (e.g., Verstraete et al. 2001; Tran et al. 2001; Peeters et al. 2002, 2004a; Förster Schreiber et al. 2004; Armus et al. 2004; Madden et al. 2006). For this work we concentrate on diagnostics that utilize the $6.2 \mu \mathrm{m}$ feature. The $6.2 \mu \mathrm{m}$ feature is the only strong infrared signature of PAHs not blended with an emission line or absorption trough, not near the wavelength edges of an IRS lowresolution module, and for which the blue and red sides of the "continuum" are easy to define. The equivalent widths of the $6.2 \mu \mathrm{m}$ feature are listed in Tables 1 and 2. The equivalent width is used in our diagnostics since the continuum at $6.2 \mu \mathrm{m}$ may 
contain emission from hot dust heated by either star formation or an AGN, in addition to a fractional contribution from stellar emission; the $6.2 \mu \mathrm{m}$ equivalent width is (indirectly) sensitive to the presence of an AGN.

\section{RESULTS}

\subsection{Optical Classifications of Nuclei}

Not all of the SINGS galaxies have an optical nuclear classification in the literature (e.g., Seyfert, LINER, starburst, etc.). Moreover, online databases such as the NASA/IPAC Extragalactic Database (NED) provide a heterogeneous source for such classifications. Thus, we turn to our own optical spectroscopy. Figure 2 displays a traditional diagnostic diagram (e.g., Baldwin et al. 1981) using optical spectroscopy and $20^{\prime \prime} \times 20^{\prime \prime}$ apertures for SINGS galactic nuclei. Note that such diagnostics were designed with ratios of lines closely spaced in wavelength to minimize the effects of extinction. Filled (open) circles in Figure 2 mark galaxies for which the literature indicates a Seyfert (LINER) nucleus; data points without circles in this diagram represent galaxies without a LINER or Seyfert classification in the literature. As alluded to in $\S 1$, the LINER classification can be complicated. Similar to what is observed for Seyfert galaxies, the optical properties of LINERs are consistent with a hard power-law spectrum. But LINER-type spectra can also be produced via winds, shocks, and cooling flows (Kauffmann et al. 2003). To further complicate the picture, "transition" objects are thought to be LINER or Seyfert galaxies with substantial contributions from normal star formation (e.g., Ho et al. 1993; González Delgado et al. 2004).

The dotted lines delineate typical starburst/AGN/LINER boundaries: [O III] $\lambda 5007 / \mathrm{H} \beta \sim 5$ and $[\mathrm{N}$ II] $26583 / \mathrm{H} \alpha \sim 0.6$ (e.g., Veilleux \& Osterbrock 1987; Armus et al. 1989). The long-dashed line traces the theoretical starburst/AGN boundary of Kewley et al. (2001), marking the maximum position in this diagram that can be obtained by pure photoionization. Objects lying above this line require an additional power source such as an AGN or shocks; objects lying below this line may still contain an AGN responsible for up to $\sim 30 \%$ of the emission-line flux ratios. The short-dashed line traces an empirical starburst/AGN boundary based on data from tens of thousands of Sloan Digital Sky Survey galaxies (Kauffmann et al. 2003). The Kauffman et al. (2003) line aims to define a boundary below which no galaxies contain an AGN. Objects lying in between the Kewley et al. (2001) and the Kauffmann et al. (2003) lines are likely to be composite AGN/starburst objects but still dominated by star formation. It is evident that the classification information available from the literature is insufficient or incomplete for a handful of SINGS nuclei; there is overall good agreement between the literature and our classifications, with a few exceptions. The literature does not indicate that the nucleus of NGC 1291 contains LINER or Seyfert activity, whereas our spectroscopic data place it squarely in the LINER category. On the other hand, NED lists NGC 1097, NGC 4321, and NGC 4552 as having LINER or Seyfert nuclei, yet our optical line ratios suggest that they are star-forming galaxies. NGC 3198, NGC 3621, and NGC 4826 appear to lie in a transitional regime between the starburst and LINER/Seyfert regions. ${ }^{14}$

Using the $\mathrm{H} \alpha / \mathrm{H} \beta$ ratio extracted from our optical spectra and a screen model for the dust distribution within a galaxy, the SINGS nuclei show modest attenuations, $\left\langle A_{V}\right\rangle \sim 1.0$ mag with

\footnotetext{
${ }^{14}$ Similar results are found using $[\mathrm{S}$ II] $\lambda \lambda 26716,6731$ and [O I $] \lambda 6300$ data in place of [N II] $\lambda 6584$ (e.g., Kewley et al. 2001).
}

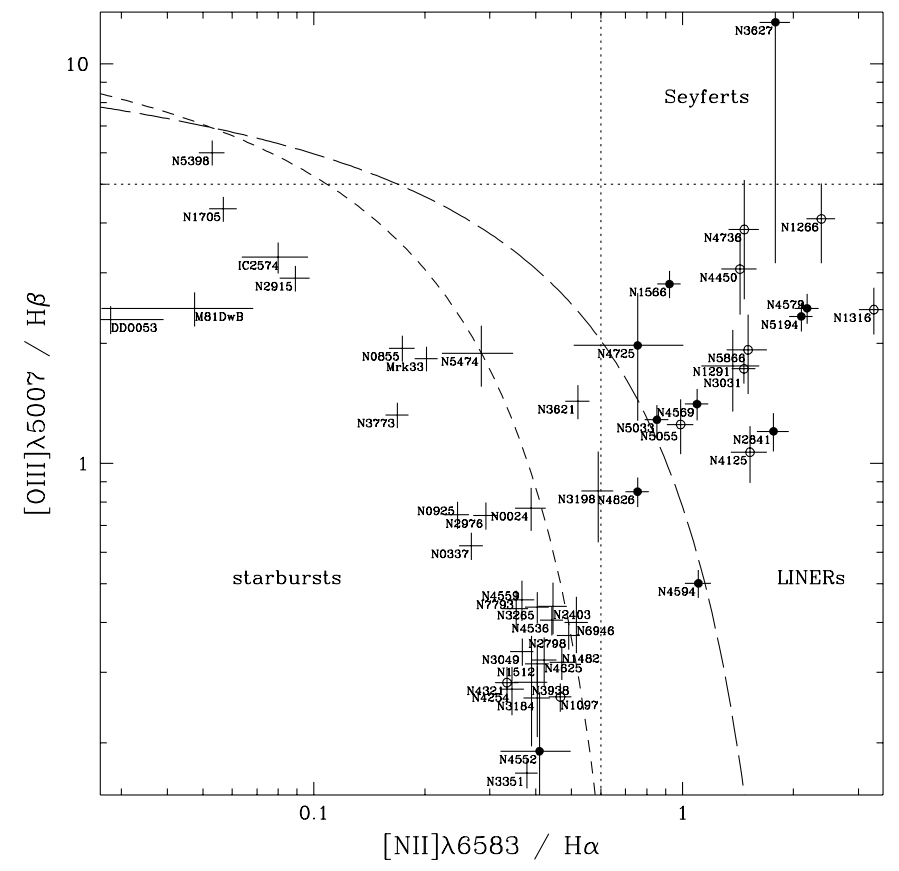

FIG. 2.-Traditional diagnostic diagram displayed for the SINGS nuclei using optical data and $20^{\prime \prime} \times 20^{\prime \prime}$ apertures (uncorrected for reddening). Filled (open) circles mark galaxies for which the literature indicates a Seyfert (LINER) nucleus. The dotted lines delineate typical starburst/Seyfert/LINER boundaries: [O III] $\lambda 5007 / \mathrm{H} \beta \sim 5$ and [ $\mathrm{N} \mathrm{II]} \lambda 6583 / \mathrm{H} \alpha \sim 0.6$ (e.g., Armus et al. 1989). The long-dashed and short-dashed lines trace the starburst/AGN boundaries of Kewley et al. (2001; theoretical) and Kauffmann et al. (2003; empirical), respectively. Error bars represent $1 \sigma$ uncertainties.

a dispersion of 1.0 mag and a maximum of $A_{V} \sim 4.1$ mag for NGC 1266. No sources appear to be heavily buried in the opti$\mathrm{cal}$, so presumably none of the classifications are skewed by large amounts of dust. ${ }^{15}$ This result is consistent with the lack of deeply buried objects in comparisons of SINGS H $\alpha$ and Spitzer $24 \mu \mathrm{m}$ data (e.g., Calzetti et al. 2005). However, as described above, there may be a few transitional objects for which the nuclear classifications are difficult to interpret in the optical. The main reason behind the analysis in this section is to provide classifications for sources that do not yet have them from the literature. Our goal is not to carry out a detailed analysis of the relative merits of classifying in the optical versus in the infrared, primarily since the SINGS sample is not optimally suited for such a test. In the next section we turn to exploring new and existing infrared diagnostics.

\subsection{Infrared Spectral Diagnostics of Nuclei and Extranuclear Regions}

\subsubsection{Emission-Line Ratios and PAH Strength}

At least $54.9 \mathrm{eV}$ is required to remove an electron from doubly ionized oxygen. On the other hand, ionizing neutral neon "only" requires $21.6 \mathrm{eV}$. Compared to $21.6 \mathrm{eV}$ photons, $54.9 \mathrm{eV}$ photons are far more likely to stem from accretion-powered disks than star formation (e.g., OB stars; Smith et al. 2004), and thus the ratio of [O IV $] 25.89 \mu \mathrm{m}$ and [ $\mathrm{Ne}$ II] $12.81 \mu \mathrm{m}$ depends on the type of source dominating the energetics of the interstellar medium. Furthermore, studies show that PAH features are quite prominent throughout much of the interstellar medium except for regions characterized by exceptionally hard radiation fields such as those

\footnotetext{
15 The exception is NGC 1377, a deeply obscured system for which the optical data are likely probing only outer layer (foreground) emission (Roussel et al. 2003; H. Roussel et al. 2006, in preparation).
} 


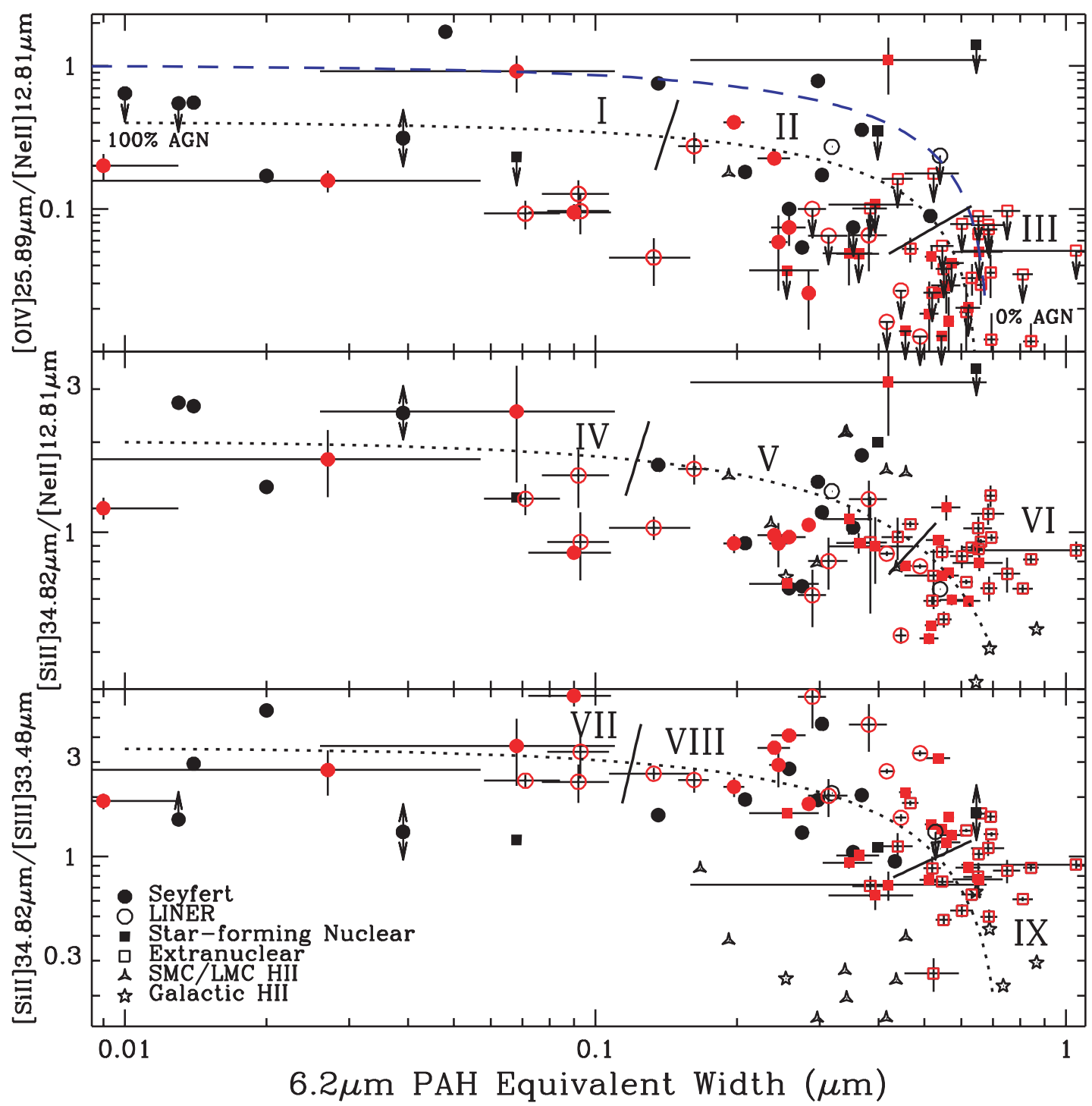

FIG. 3.-Ratios of mid-infrared forbidden lines as a function of the $6.2 \mu \mathrm{m}$ PAH feature equivalent width. SINGS data are displayed in red with $1 \sigma$ error bars based on the statistical uncertainties; archival data without error bars are indicated with black symbols and described in $\S 3.2$. The dotted lines are linear mixing models of a "pure" AGN and a "pure" star-forming source (see text). The dashed line in the top panel is a similar mixing model first presented by Genzel et al. (1998). The solid lines and Roman numerals delineate regions distinguished by Seyfert galaxies, LINERs, star formation, etc. (see Table 4 and $\S$ 5.2.1).

that arise in AGNs and the cores of $\mathrm{H}$ il regions (Cesarsky et al. 1996; Sturm et al. 2000). A mid-infrared diagnostic diagram first put forth by Genzel et al. (1998) and later explored by Peeters et al. (2004b) plots the emission-line ratio [O IV] $25.89 \mu \mathrm{m} /[\mathrm{Ne}$ II] $12.81 \mu \mathrm{m}$ versus the strength of a mid-infrared PAH feature. In such plots AGN sources show enhanced [O Iv] $25.89 \mu \mathrm{m}$ emission and comparatively weak PAH feature strength. However, those two studies focused on AGN-dominated, ULIRG, and starburst systems. The top panel of Figure 3 uses the $6.2 \mu \mathrm{m}$ PAH feature and the emission-line ratio $[\mathrm{O}$ IV $] 25.89 \mu \mathrm{m} /[\mathrm{Ne}$ II] $12.81 \mu \mathrm{m}$ in a similar mid-infrared diagnostic diagram, but one that incorporates "normal" (starbursting/star-forming) nuclei and $\mathrm{H}$ II regions: the sensitivity of Spitzer allows us to probe to far fainter levels than heretofore possible. ${ }^{16}$ As expected, normal nuclei and $\mathrm{H}$ II regions extend the previously observed trend: lower luminosity star-forming nuclei and $\mathrm{H}$ II regions exhibit comparatively large $6.2 \mu \mathrm{m}$ equivalent widths and relatively low ratios

\footnotetext{
${ }^{16}$ Additional high-ionization line possibilities include [ $\left.\mathrm{Nev}\right] 14.32 \mu \mathrm{m}$ and [S Iv] $10.51 \mu \mathrm{m}$, but these are less frequently detected than $\left[\mathrm{O}_{\mathrm{Iv}}\right] 25.89 \mu \mathrm{m}$ in SINGS spectra.
}

of [O IV $] 25.89 \mu \mathrm{m} /[\mathrm{Ne}$ II] $12.81 \mu \mathrm{m}$, indicating strong contributions from [ $\mathrm{Ne}$ II] $12.81 \mu \mathrm{m}$ cooling of $\mathrm{H}$ II regions and their PAH-rich photodissociation region surroundings and negligible emission from AGNs.

High-ionization lines like [O Iv] $25.89 \mu \mathrm{m}$ are somewhat difficult to detect in many SINGS sources. Two alternative diagnostic diagrams are also provided in Figure 3. Both the middle and bottom panels involve the more easily detectable [Si II] $34.82 \mu \mathrm{m}$ line, which has an ionization potential of $8.15 \mathrm{eV}$. The normalization for the [Si II] $34.82 \mu \mathrm{m}$ line in the middle and bottom panels utilizes strong mid-infrared lines with similar ionization potentials: [ $\mathrm{Ne}$ II] $12.81 \mu \mathrm{m}(21.6 \mathrm{eV})$ and [S III] $33.48 \mu \mathrm{m}(23.3 \mathrm{eV})$. An advantage to using [ $\mathrm{Ne}$ II] $12.81 \mu \mathrm{m}$ as the normalization is that it lies in the much less noisy ShortHigh module of IRS. Conversely, the [S III] $33.48 \mu$ m line lies in the same Long-High module as the [Si II] $34.82 \mu \mathrm{m}$ line, which can improve observing efficiency and minimize cross module uncertainties involving calibration and aperture matching. In addition, the short-wavelength baseline between the [Si II] $34.82 \mu \mathrm{m}$ and [S III] $33.48 \mu \mathrm{m}$ lines minimizes the effects of extinction. 
TABLE 4

Classifications by Region in Figure 3

\begin{tabular}{|c|c|c|c|c|c|}
\hline Region & Number of Sources & $\begin{array}{l}\text { Seyfert } \\
(\%)\end{array}$ & $\begin{array}{l}\text { LINER } \\
(\%)\end{array}$ & $\begin{array}{c}\text { H II Nuclei } \\
(\%)\end{array}$ & $\begin{array}{c}\text { Extranuclear }+ \text { H II Regions } \\
(\%)\end{array}$ \\
\hline I & 15 & 73 & 20 & 7 & 0 \\
\hline II & 31 & 42 & 23 & 19 & 16 \\
\hline 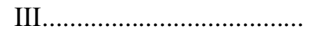 & 31 & 0 & 10 & 32 & 58 \\
\hline IV & 12 & 67 & 25 & 8 & 0 \\
\hline V & 39 & 33 & 18 & 21 & 28 \\
\hline 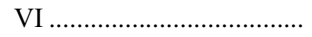 & 34 & 0 & 9 & 26 & 65 \\
\hline 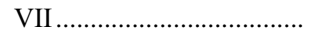 & 12 & 67 & 25 & 8 & 0 \\
\hline 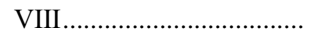 & 45 & 31 & 24 & 27 & 18 \\
\hline IX & 31 & 0 & 0 & 16 & 84 \\
\hline
\end{tabular}

Note.- “Extranuclear + H II regions" implies SINGS extranuclear/H II regions in addition to Milky Way and Magellanic Cloud H II regions.

Why are similar trends seen in the three panels? The answer may lie in the physics of X-ray-dominated regions. As pointed out by Maloney et al. (1996), the [Si II] $34.82 \mu \mathrm{m}$ line is a strong coolant of X-ray-irradiated gas. In X-ray-dominated regions around AGNs, the [ $\mathrm{Si}$ II] emission dominates that from the comparatively small $\mathrm{H}$ II-like regions surrounding the hard-spectrum source. Moreover, X-ray-dominated regions can be quite large since hard X-ray photons penetrate large column densities, and the conversion of X-ray energy to infrared continuum and line emission can be very efficient. Maloney et al. (1996) predict that [Si II] $34.82 \mu \mathrm{m}$, [O I $63 \mu \mathrm{m}$, [C II] $158 \mu \mathrm{m}$, and [C I] $609 \mu \mathrm{m}$ are the top four cooling lines within X-ray-dominated regions, with [Si II] $34.82 \mu \mathrm{m}$ having an amplitude $1 \%-10 \%$ that of the far-infrared luminosity for an extremely large range of physical conditions.

An argument based on interstellar density provides another possibility for the high [Si II] $34.82 \mu \mathrm{m} /[\mathrm{Ne}$ II] $12.81 \mu \mathrm{m}$ and [Si II] $34.82 \mu \mathrm{m} /[\mathrm{S}$ III] $33.48 \mu \mathrm{m}$ ratios in AGNs. Kaufman et al. (2006) show that the ratio $[\mathrm{Si}$ II](PDR)/[Si II](H II) increases with increasing density. In fact, for low-density $\mathrm{H}$ II regions most of the [Si II] comes from the $\mathrm{H}$ II region and not the surrounding photodissociation region. Moreover, Meijerink \& Spaans (2005) show that the ratio [Si II] (XDR)/[Si II] (PDR) also increases with increasing density. AGNs may have their emitting gas at higher densities than typically found in starbursts and normal galaxies, leading to increased [Si II] $34.82 \mu \mathrm{m} /\left[\mathrm{Ne}_{\mathrm{II}}\right] 12.81 \mu \mathrm{m}$ and [Si II] $34.82 \mu \mathrm{m} /[\mathrm{S}$ III] $33.48 \mu \mathrm{m}$ ratios. In other words, the prominent [Si II] $34.82 \mu \mathrm{m}$ line for AGN sources may be due to strong [Si II] cooling of X-ray-dominated regions or enhanced [Si II] emission from the surrounding dense photodissociation regions.

A third scenario for enhanced [Si II] $34.82 \mu \mathrm{m}$ in AGNs involves the extent to which silicon is depleted onto dust grains. Heavy elements such as $\mathrm{Si}, \mathrm{Mg}$, and $\mathrm{Fe}$ may be returned to the gas phase by dust destruction (e.g., sputtering) in regions subject to strong shocks caused by stellar winds, starbursts, and AGN activity. So perhaps (gas phase) silicon lines are stronger in active galaxies due to this effect.

If the strong [Si II] $34.82 \mu \mathrm{m}$ emission is due to the cooling of X-ray-dominated regions, it should be noted that relatively strong low-ionization line emission (e.g., [O I] 26300 and [O I] $63 \mu \mathrm{m}$ ) has previously been observed emanating from the large "partially ionized regions" surrounding AGNs and infrared-bright galaxies (Veilleux \& Osterbrock 1987; Armus et al. 1989; Veilleux 1991; Spinoglio \& Malkan 1992; Osterbrock 1993; Dale et al. 2004a). Hence, strong low-ionization line emission from AGNs is not a new concept. We take advantage of this concept to present new techniques for distinguishing between AGN sources and star-forming regions. These techniques rely on an easily detect- able, prominent cooling line of a low-ionization species associated with X-ray-dominated regions, the dense interstellar material illuminated by power-law radiation fields.

The regions of Figure 3 where Seyfert galaxies/LINERs/ starbursts mix are quite large, although the bottom panel perhaps shows a cleaner separation (less mixing) between Seyfert galaxies+LINERs and star-forming regions; only the top left and bottom right extremes allow for a clean separation between classifications. Short solid lines roughly perpendicular to the dotted AGN/star-forming lines delineate three regions in the panels of Figure 3. The boundaries are

$$
\begin{aligned}
& \log \left(\frac{[\mathrm{O} \text { Iv }] 25.89 \mu \mathrm{m}}{[\mathrm{Ne} \mathrm{II}] 12.81 \mu \mathrm{m}}\right)=10 \log [\mathrm{EW}(6.2 \mu \mathrm{m} \mathrm{PAH})]+8.0, \\
& \log \left(\frac{[\mathrm{O} \text { IV }] 25.89 \mu \mathrm{m}}{\left[\mathrm{Ne}_{\mathrm{II}}\right] 12.81 \mu \mathrm{m}}\right)=1.9 \log [\mathrm{EW}(6.2 \mu \mathrm{m} \mathrm{PAH})]-0.6, \\
& \log \left(\frac{[\mathrm{Si} \mathrm{II}] 34.82 \mu \mathrm{m}}{[\mathrm{Ne} \mathrm{II}] 12.81 \mu \mathrm{m}}\right)=5.0 \log [\mathrm{EW}(6.2 \mu \mathrm{m} \mathrm{PAH})]+4.8, \\
& \log \left(\frac{[\mathrm{Si}}{[\mathrm{II}] 34.82 \mu \mathrm{m}}\right)=1.7 \log [\mathrm{EW}(6.2 \mu \mathrm{m} \mathrm{PAH})]+0.5, \\
& \log \left(\frac{[\mathrm{Si} \mathrm{II}] 34.82 \mu \mathrm{m}}{[\mathrm{S} \mathrm{IIII}] 33.48 \mu \mathrm{m}}\right)=10 \log [\mathrm{EW}(6.2 \mu \mathrm{m} \mathrm{PAH})]+9.7, \\
& \log \left(\frac{[\mathrm{Si} \mathrm{II}] 34.82 \mu \mathrm{m}}{[\mathrm{S} \mathrm{III}] 33.48 \mu \mathrm{m}}\right)=1.1 \log [\mathrm{EW}(6.2 \mu \mathrm{m} \mathrm{PAH})]+0.3
\end{aligned}
$$

for regions I-II, II-III, IV-V, V-VI, VII-VIII, and VIII-IX, respectively. The population statistics for these regions, provided in Table 4, show that regions I+IV+VII and III+VI+IX are representative (at the $>90 \%$ level) of Seyfert galaxies/LINERs and star-forming systems, respectively. Regions II $+\mathrm{V}+\mathrm{VIII}$, on the other hand, contain a mix of classifications and thus represent transition regions: either the source classifications in these regions are ambiguous or the regions simply contain a more heterogeneous mixture of pure types. Seyfert nuclei could shift toward the location of star-forming nuclei due to aperture effects: although the same solid angles are used for extracting the line data, the range of distances in the sample leads to a range in physical apertures. Conversely, some star-forming nuclei exhibit relatively large line ratios and small PAH equivalent widths in Figure 3. Perhaps a fraction of the star-forming regions contain significant numbers of Wolf-Rayet stars, leading to enhanced [O Iv] emission (e.g., Schaerer \& Stasińska 1999). Further, maybe a decreased PAH equivalent width results from a relatively low 


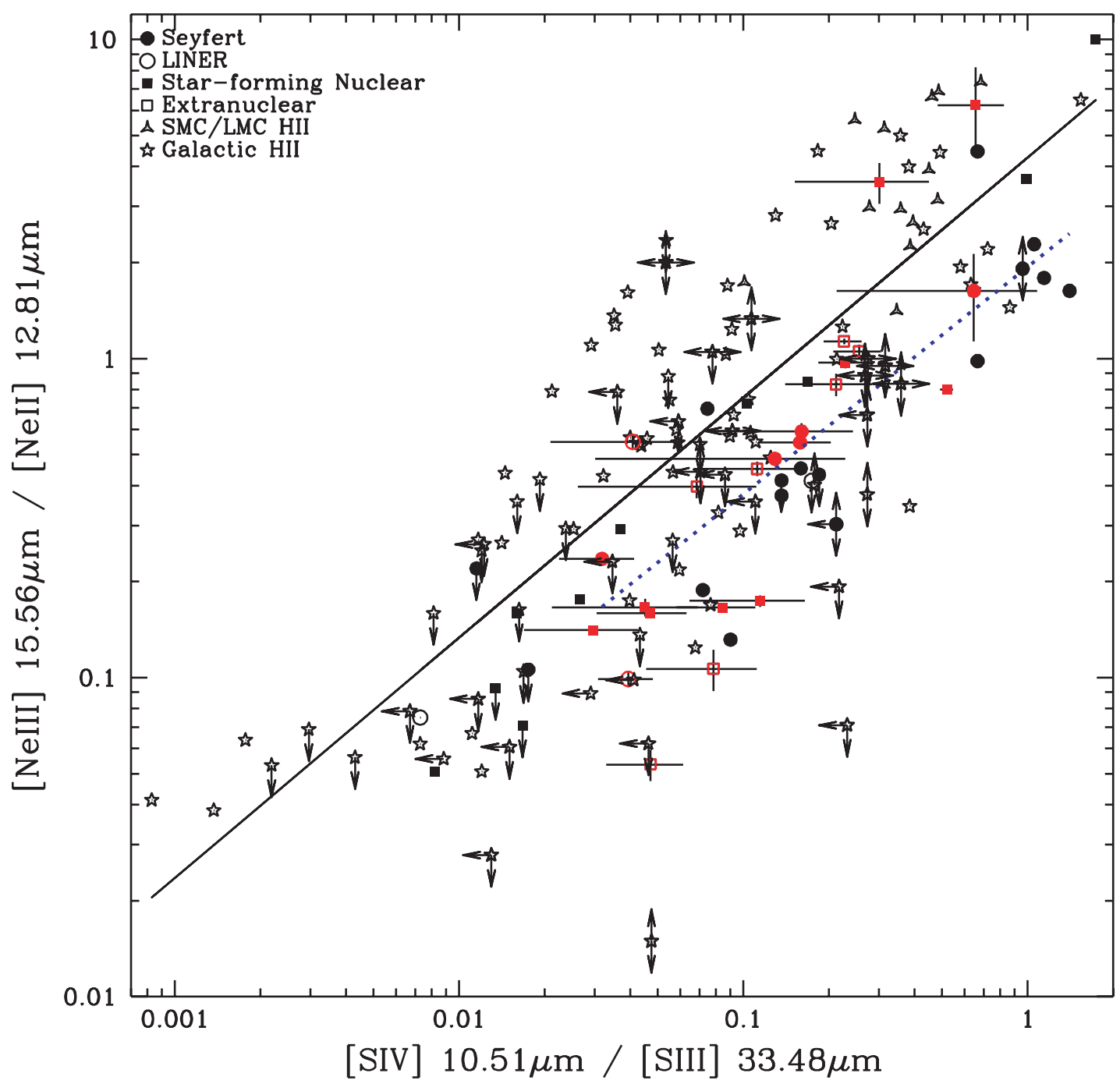

FIG. 4.-Diagnostic diagram involving ratios of neon and sulfur lines at different ionization levels (see, e.g., Verma et al. 2003). The data are displayed as described in Fig. 3. The solid line is a linear fit to the detections of star-forming nuclei and $\mathrm{H}$ II regions, while the dotted line is a linear fit to the Seyfert detections.

ratio of photodissociation region to $\mathrm{H}$ II region contributions (Laurent et al. 2000).

The dotted lines in Figure 3 represent a variable mix of an AGN nucleus and a "pure" star-forming region. The anchors for these mixing models are the following:

$$
\mathrm{EW}(6.2 \mu \mathrm{m} \mathrm{PAH}) \approx 0.01 \mu \mathrm{m},
$$

$$
\begin{gathered}
\frac{[\mathrm{O} \text { IV }] 25.89 \mu \mathrm{m}}{[\mathrm{Ne} \text { II }] 12.81 \mu \mathrm{m}} \approx 0.4 \quad(100 \% \mathrm{AGN}), \\
\mathrm{EW}(6.2 \mu \mathrm{m} \mathrm{PAH}) \approx 0.7 \mu \mathrm{m}, \\
\frac{[\mathrm{O} \text { IV }] 25.89 \mu \mathrm{m}}{[\mathrm{Ne} \text { II }] 12.81 \mu \mathrm{m}} \approx 0.01 \quad(100 \% \mathrm{H} \mathrm{II}), \\
\mathrm{EW}(6.2 \mu \mathrm{m} \mathrm{PAH}) \approx 0.01 \mu \mathrm{m}, \\
\frac{[\mathrm{Si} \text { II }] 34.82 \mu \mathrm{m}}{[\mathrm{Ne} \text { II }] 12.81 \mu \mathrm{m}} \approx 2 \quad(100 \% \mathrm{AGN}), \\
\mathrm{EW}(6.2 \mu \mathrm{m} \mathrm{PAH}) \approx 0.7 \mu \mathrm{m}, \\
\frac{[\mathrm{Si} \text { II }] 34.82 \mu \mathrm{m}}{[\mathrm{Ne} \text { II }] 12.81 \mu \mathrm{m}} \approx 0.4 \quad(100 \% \mathrm{H} \mathrm{II}),
\end{gathered}
$$

$\mathrm{EW}(6.2 \mu \mathrm{m} \mathrm{PAH}) \approx 0.01 \mu \mathrm{m}$,

$$
\begin{aligned}
& \frac{[\mathrm{Si} \text { II }] 34.82 \mu \mathrm{m}}{[\mathrm{S} \text { III }] 33.48 \mu \mathrm{m}} \approx 3.5 \quad(100 \% \mathrm{AGN}) \text {, } \\
& \operatorname{EW}(6.2 \mu \mathrm{m} \mathrm{PAH}) \approx 0.7 \mu \mathrm{m}, \\
& \frac{[\mathrm{Si} \text { II }] 34.82 \mu \mathrm{m}}{[\mathrm{S} \text { III }] 33.48 \mu \mathrm{m}} \approx 0.2 \quad(100 \% \mathrm{H} \mathrm{II}) \text {. }
\end{aligned}
$$

The dashed line in the top panel of Figure 3 shows the approximate mixing model of Genzel et al. (1998, their Fig. 5), obtained after empirically deriving a relation between their $7.7 \mu \mathrm{m} \mathrm{PAH}$ "strength" (line-to-continuum ratio) and the $6.2 \mu \mathrm{m}$ PAH equivalent width. Many of the high-ionization line data presented by Genzel et al. (1998) were upper limits, so it is unsurprising that their original line lies above our line (although the discrepancy may also lie in small number statistics).

\subsubsection{Line Ratios of Different Ionization States of the Same Element}

Figure 4 plots a ratio of doubly to singly ionized neon as a function of a ratio of triply to doubly ionized sulfur (see also Verma et al. 2003). Many of the data points in this plot are for 


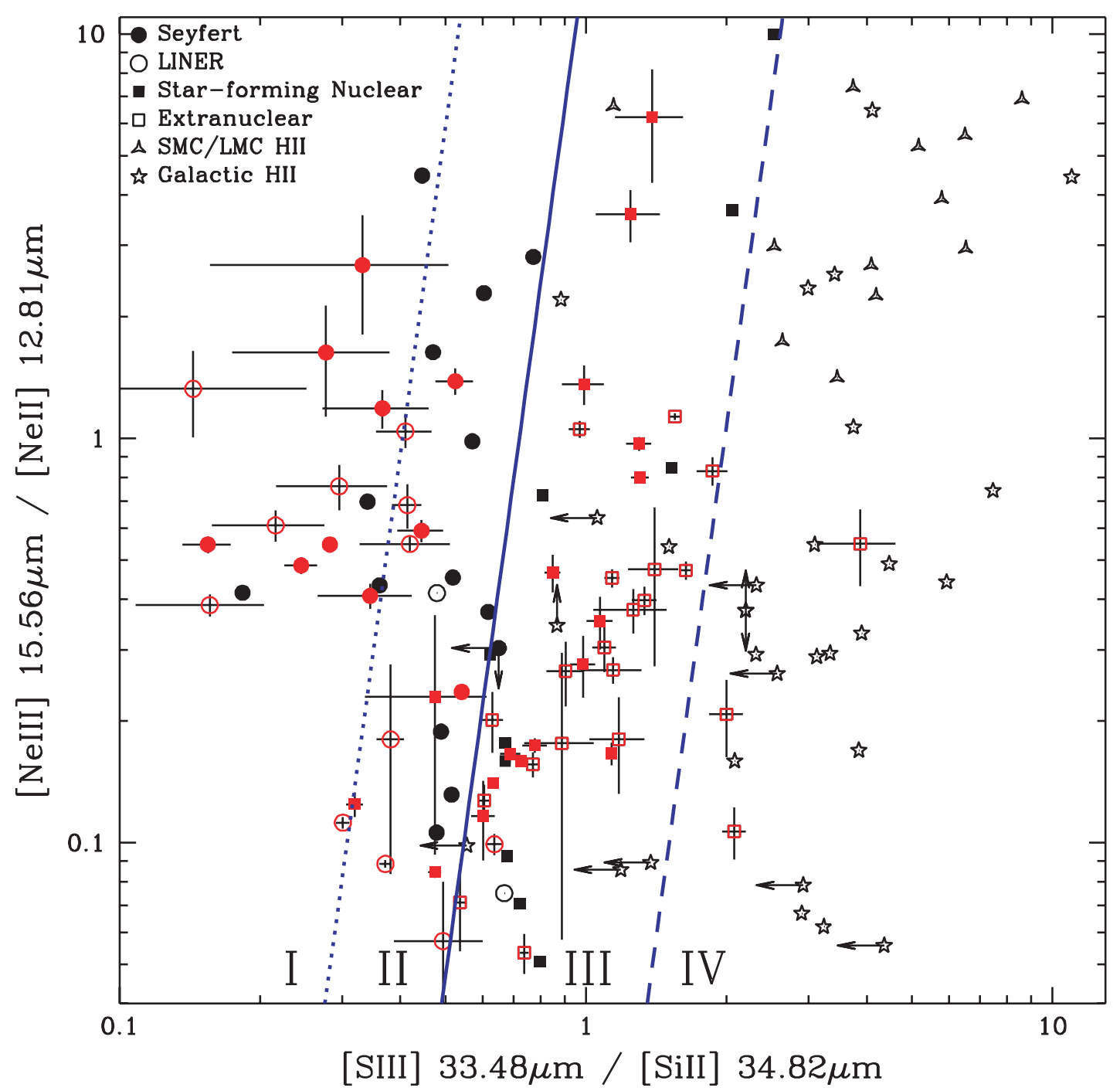

Fig. 5.- Neon, sulfur, and silicon diagnostic diagram involving ratios of lines at different ionizations. The data are displayed as described in Fig. 3. The lines and Roman numerals delineate regions distinguished by Seyfert galaxies, LINERs, star formation, etc. (see Table 5 and $\S 5.2 .3$ ).

Galactic H II regions (Vermeij et al. 2002; Giveon et al. 2002; Peeters et al. 2002; see $\S 3.2$ ). Clearly the neon excitation tracks the sulfur excitation. To first order, there does not appear to be any sequence in the distribution according to source classification (Seyfert, starburst, H II, etc.). However, the low-metallicity $\mathrm{H}$ II regions from the Magellanic Clouds are preferentially in the high-excitation, upper right corner of the diagram. Presumably the diminished line blanketing for low-metallicity sources results in a harder radiation field and thus higher excitations (see Genzel \& Cesarsky 2000; Martín-Hernández et al. 2002; Madden et al. 2006). In addition, AGN sources show somewhat lower [Ne III] $15.6 \mu \mathrm{m} /[\mathrm{Ne}$ II] $12.81 \mu \mathrm{m}$ ratios than exhibited by starforming sources, and the locus of the AGN detections lies at slightly higher values of [S IV] $10.51 \mu \mathrm{m} /[\mathrm{S}$ III] $33.48 \mu \mathrm{m}$. The

TABLE 5

Classifications by Region in Figure 5

\begin{tabular}{|c|c|c|c|c|c|}
\hline Region & Number of Detections & $\begin{array}{l}\text { Seyfert } \\
(\%)\end{array}$ & $\begin{array}{l}\text { LINER } \\
(\%)\end{array}$ & $\begin{array}{c}\text { H II Nuclei } \\
(\%)\end{array}$ & $\begin{array}{c}\text { Extranuclear }+\mathrm{H} \text { II Regions } \\
(\%)\end{array}$ \\
\hline I+II & 38 & 61 & 32 & 8 & 0 \\
\hline 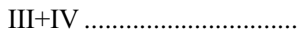 & 79 & 0 & 3 & 30 & 67 \\
\hline I & 16 & 69 & 31 & 0 & 0 \\
\hline II & 22 & 55 & 32 & 14 & 0 \\
\hline 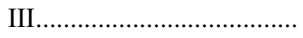 & 47 & 0 & 4 & 51 & 45 \\
\hline 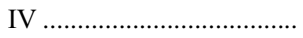 & 32 & 0 & 0 & 0 & 100 \\
\hline
\end{tabular}

Note._- "Extranuclear + H II regions" implies SINGS extranuclear/H II regions in addition to Milky Way and Magellanic Cloud H II regions. 
dotted and solid lines show linear fits to the Seyfert and starforming sources and have slopes of $0.71( \pm 0.12)$ and $0.75( \pm 0.06)$, respectively.

\subsubsection{A Neon, Sulfur, and Silicon Diagnostic}

If the neon excitation is plotted as a function of [S III] $33.48 \mu \mathrm{m} /$ [Si II] $34.82 \mu \mathrm{m}$ (Fig. 5), a more obvious separation of the starforming and AGN-powered data points is observed. Not only do the low-metallicity Magellanic Cloud regions exhibit a higher neon excitation, but nearly all of the "pure star-forming" nuclei and extranuclear regions show relatively elevated ratios in [S $\mathrm{S}_{\text {III }} 33.48 \mu \mathrm{m} /\left[\mathrm{Si}_{\text {III }} 34.82 \mu \mathrm{m}\right.$ (see also Fig. 3). Note in addition that many of the filled squares representing starbursting/ star-forming nuclei are located between the $\mathrm{H}$ II regions and the AGNs. Table 5 quantifies the source type fractions within each of the four regions delineated by the lines drawn in Figure 5. The boundaries are defined by lines with the same slope but differing offsets:

$$
\log \left(\frac{[\mathrm{Ne} \text { III] } 15.56 \mu \mathrm{m}}{[\mathrm{Ne} \text { II }] 12.81 \mu \mathrm{m}}\right)=8.4 \log \left(\frac{[\mathrm{S} \text { III] } 33.48 \mu \mathrm{m}}{\left[\mathrm{Si}_{\mathrm{II}}\right] 34.82 \mu \mathrm{m}}\right)+\gamma,
$$

where $\gamma=(+3.3,+1.2,-2.5)$ for the lines demarcating regions (I-II, II-III, III-IV).

The numbers provided in Table 5 can be used to determine the statistical reliability of a classification for a galaxy randomly drawn from a mid-infrared line survey. For example, if a galaxy appears in region III or region IV, it should be classified as a starforming system with a $1 \sigma$ confidence interval of $84 \%-93 \%$ or $91 \%-98 \%$, respectively. Likewise, a galaxy residing in region I or region II should be classified as an AGN powered with a $1 \sigma$ confidence interval of $83 \%-97 \%$ or $73 \%-88 \%$, respectively.

These results can be partially understood in the context of the cooling line physics introduced above. The [Si II] $34.82 \mu \mathrm{m}$ line is a significant coolant of X-ray-ionized regions or dense photodissociation regions (Hollenbach \& Tielens 1999), whereas the [S III] $33.48 \mu \mathrm{m}$ line is a strong marker of $\mathrm{H}$ II regions. In other words, extranuclear regions and star-forming nuclei will show strong signatures of the Strömgren sphere coolant [S III] $33.48 \mu \mathrm{m}$, while AGNs and their associated X-ray-dominated regions or dense photodissociation regions will exhibit relatively strong [Si II] $34.82 \mu \mathrm{m}$ emission in analogy to the increased strength of [O I] $\lambda 6300$ emission in AGNs (e.g., Veilleux \& Osterbrock 1987). In addition, the fraction of photodissociation regions falling within each beam will play a role in the line ratios. The data for Magellanic Cloud and Galactic $\mathrm{H}$ II regions stem from smaller physical apertures and thus are likely to have fractionally higher contributions from Strömgren spheres than photodissociation regions.

Metallicity may be a factor as well. Since the central regions of galaxies typically are more abundant in heavy metals (Pagel \& Edmunds 1981; McCall 1982; Vila-Costas \& Edmunds 1992; Pilyugin \& Ferrini 1998; Henry \& Worthey 1999) and, as explained above, a lower metallicity can lead to harder radiation fields and thus enhanced high-ionization-to-low-ionization line ratios, it is possible that this $\mathrm{AGN} \rightarrow \mathrm{H}$ II nucleus $\rightarrow \mathrm{H}$ II region sequencing along the [S III] $33.48 \mu \mathrm{m} /[\mathrm{Si}$ II $] 34.82 \mu \mathrm{m}$ axis is affected by metallicity. However, the lower metallicity Magellanic Cloud data are not substantially to the right of the Galactic $\mathrm{H}$ II region data, so the effect is not solely due to metallicity. Alternatively, perhaps some of the star-forming

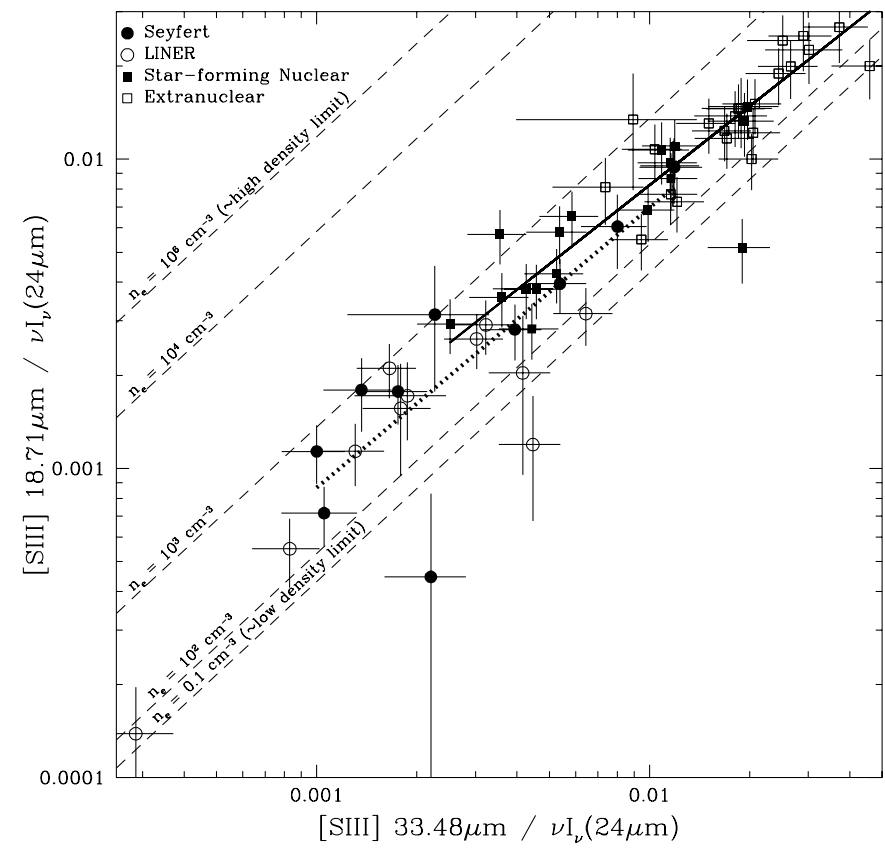

FIG. 6.- Correlation between two transitions of doubly ionized sulfur, normalized by the flux at $24 \mu \mathrm{m}$. This plot includes only SINGS data and $1 \sigma$ error bars. The solid line is a linear fit to the detections of star-forming nuclei and $\mathrm{H}$ II regions, while the dotted line is a linear fit to the Seyfert detections. The set of dashed lines represent different constant interstellar electron densities. Most of the SINGS data are bounded by the low- and high-density limiting values, and several are consistent with the low-density limiting value of $[\mathrm{S} \mathrm{III]} 18.71 \mu \mathrm{m} /[\mathrm{S}$ III] $33.48 \mu \mathrm{m}=0.43$ at $0.1 \mathrm{~cm}^{-3}$. The SINGS data typically exhibit $n_{e} \sim 400 \mathrm{~cm}^{-3}$ [See the electronic edition of the Journal for a color version of this figure.]

nuclei have contributions from undetected weak AGNs and thus are not "pure" star-forming nuclei, resulting in a location for star-forming nuclei on this diagram between AGNs and $\mathrm{H}$ II regions.

\subsubsection{Density Diagnostics}

The average line ratio of [S III] $18.71 \mu \mathrm{m}$ to [S III] $33.48 \mu \mathrm{m}$ for the SINGS sample is 0.82 with a $1 \sigma$ dispersion of 0.27 . This ratio implies an interstellar electron density of $\left\langle n_{e}\right\rangle \sim 400_{-290}^{+240} \mathrm{~cm}^{-3}$ for the $\sim 23^{\prime \prime} \times 15^{\prime \prime}$ nuclear and extranuclear regions of SINGS galaxies. The average density is calculated using electron collision strengths from Tayal \& Gupta (1999) and excluding the effects of differential extinction at these mid-infrared wavelengths (which is shown in $\S 5.1$ to be relatively small at optical wavelengths). This density on roughly kiloparsec scales is typical of starburst/ LINER/Seyfert galaxies (Kewley et al. 2001) but lower than the $\sim 10^{3}-10^{4} \mathrm{~cm}^{-3}$ found for high surface brightness $\mathrm{H}$ II regions using small apertures uncontaminated by the surrounding neutral interstellar medium and lower density $\mathrm{H}$ II regions (e.g., Wang et al. 2004). A visual way to explore this doubly ionized sulfur line ratio is portrayed in Figure 6 using the aperturematched $24 \mu \mathrm{m}$ flux as a normalization for the line fluxes. The set of dashed lines represent different interstellar electron densities. The correlation in Figure 6 extends over 2 orders of magnitude in the line-to-continuum ratios and encompasses both AGN-dominated and star formation-dominated sources; a nonparametric ranking analysis indicates a global correlation at the $7 \sigma$ level. Linear fits to the two separate star-forming and Seyfert populations emphasize that, although the trends for the two populations are similar, the nuclei with Seyfert characteristics (dotted line; slope $0.91 \pm 0.22$ ) differ along the diagonal from the starbursting nuclei and the $\mathrm{H}$ II regions (solid line; slope $0.85 \pm 0.05$ ). 
Star-forming sources show more pronounced [S III] $33.48 \mu \mathrm{m}-$ to-continuum ratios compared to Seyfert galaxies and LINERs, consistent with the notion that [S III] $33.48 \mu \mathrm{m}$ is an important coolant of $\mathrm{H}$ II regions. In addition, we are seeing the effects of continuum dilution in the line-to-continuum ratio for Seyfert galaxies and LINERs, sources for which hot dust emission is pronounced in the mid-infrared and thus the line-to-continuum ratios are suppressed (e.g., Laurent et al. 2000; Dale et al. 2001; Xu et al. 2001; Siebenmorgen et al. 2004). Finally, similar to what is seen in Figures 3 and 5, but perhaps not as prominently, the data in Figure 6 suggest that the $\mathrm{H}$ II regions (open squares) occupy a different portion of the diagram than star-forming nuclei ( filled squares). $\mathrm{H}$ II regions have higher line-to-continuum ratios than star-forming nuclei, which in turn have higher ratios than Seyfert galaxies and LINERs.

\section{SUMMARY}

We have presented mid-infrared diagnostic diagrams for a large portion of the SINGS sample supplemented by archival ISO and Spitzer data. A portion of our work solidifies and extends previous $I S O$-based mid-infrared work to lower luminosity normal galactic nuclei and H II regions using the Spitzer Space Telescope. We also present new diagnostics that effectively constrain a galaxy's dominant power source. The power of the diagnostic diagrams of Genzel et al. (1998; see also Peeters et al. 2004b) for distinguishing between AGNs and star-forming sources in dusty ULIRGs is that mid-infrared lines and PAH features are much less affected by extinction than their optical counterparts in a traditional diagnostic diagram. Unlike diagrams put forth by Genzel et al. (1998), which rely on detecting relatively weak highionization lines like [O Iv] $25.89 \mu \mathrm{m}$ and [ Ne v] $14.32 \mu \mathrm{m}$, we provide a new diagnostic that utilizes a strong low-ionization line. The advantage of using a line ratio like $\left[\mathrm{Si}_{\mathrm{II}}\right] /\left[\mathrm{Ne} \mathrm{II}_{\mathrm{II}}\right]$ is that singly ionized silicon and neon have ionization potentials of only 8.15 and $12.6 \mathrm{eV}$, respectively, so they can both be observed over a large range of physical conditions. This is similar in concept to previous efforts that have taken advantage of $\left[\mathrm{O}_{\mathrm{I}}\right]$ lines (e.g., at $6300 \AA$ or $63 \mu \mathrm{m})$ that are coolants of the X-ray-dominated regions (or dense photodissociation regions) surrounding AGNs. In plots of $[\mathrm{O} \mathrm{IV}] /[\mathrm{Ne} \mathrm{II}],[\mathrm{Si} \mathrm{II}] /[\mathrm{Ne} \mathrm{II}]$, and $[\mathrm{Si} \mathrm{II}] /[\mathrm{S}$ III] versus $6.2 \mu \mathrm{m}$ PAH equivalent width, we identify regions where $>90 \%$ of the sources are Seyfert or LINER. Likewise, additional regions in all three plots show populations comprised of more than $90 \% \mathrm{H}$ II regions or star-forming nuclei.

Another useful mid-infrared diagnostic is [ $\mathrm{Ne}$ III] $15.56 \mu \mathrm{m} /$ [ $\mathrm{Ne}$ II] $12.81 \mu \mathrm{m}$ versus [S III] $33.48 \mu \mathrm{m} /[\mathrm{Si}$ II] $34.82 \mu \mathrm{m}$. This plot tracks the excitation power of the radiation field on one axis, while the other axis is a relative measure of the cooling of $\mathrm{H}$ II regions and X-ray-dominated regions (or dense photodis- sociation regions). Similar to what is found for the diagnostics mentioned above, both starbursting nuclei and extranuclear regions stand apart from nuclei that are powered by accretionpowered disks. Moreover, compared to starbursting nuclei, extranuclear regions typically separate even further from Seyfert nuclei, especially for low-metallicity environments. Presumably this extranuclear $\rightarrow$ nuclear separation occurs since extranuclear regions are cleaner representatives of $\mathrm{H}$ II regions than starburst nuclei because their stellar populations and interstellar medium structure are less complex. Extranuclear regions more likely contain younger stellar populations since they trace a single burst, as opposed to the average of multiple star formation episodes for nuclei (e.g., Dale et al. 2004b). Finally, we note that it is difficult to clearly distinguish between pure Seyfert and pure LINER sources using these diagnostics.

The line ratio [S $\left.\mathrm{S}_{\mathrm{III}}\right] 18.71 \mu \mathrm{m} /[\mathrm{S}$ III] $33.48 \mu \mathrm{m}$ yields an average interstellar electron density of $\left\langle n_{e}\right\rangle \sim 400_{-290}^{+240} \mathrm{~cm}^{-3}$ for the $\sim 23^{\prime \prime} \times 15^{\prime \prime}$ nuclear and extranuclear regions of SINGS galaxies. This density is much closer (in log space) to the theoretical low-density limit of Tayal \& Gupta (1999) than their high-density limit, and in fact the data for several sources are consistent with the low-density limiting value. In addition to the interstellar gas densities being unremarkable, there are no SINGS sources sufficiently obscured by dust such that optical and infrared diagnostics provide obviously discrepant classifications of the energy source. This is not surprising, however, since our nuclei exhibit modest extinctions, $\left\langle A_{V}\right\rangle \sim 1.0 \mathrm{mag}$, and normal starforming galactic nuclei in general show $0 \mathrm{mag} \lesssim A_{V} \lesssim 3 \mathrm{mag}$ (Ho et al. 1997a). This relative transparency means that the SINGS sample is not ideally suited for a detailed comparison of the relative merits of optical and infrared classifications. However, the diverse SINGS sample of nuclear and extranuclear regions provides an enormous range of physical parameters, and this has proved critical in developing the mid-infrared diagnostics in this paper.

Mike Brotherton provided helpful comments. Support for this work, part of the Spitzer Space Telescope Legacy Science Program, was provided by NASA through contract 1224769 issued by the Jet Propulsion Laboratory, California Institute of Technology under NASA contract 1407 . This research has made use of the NASA/IPAC Extragalactic Database, which is operated by JPL/Caltech, under contract with NASA. This publication makes use of data products from the Two Micron All Sky Survey, which is a joint project of the University of Massachusetts and the Infrared Processing and Analysis Center/California Institute of Technology, funded by the National Aeronautics and Space Administration and the National Science Foundation.
Armus, L., Heckman, T. M., \& Miley, G. K. 1989, ApJ, 347, 727

Armus, L., et al. 2004, ApJS, 154, 178

Baldwin, J. A., Phillips, M. M., \& Terlevich, R. 1981, PASP, 93, 5

Calzetti, D., et al. 2005, ApJ, 633, 871

Cesarsky, D., Lequeux, J., Abergel, A., Perault, M., Palazzi, E., Madden, S., \& Tran, D. 1996, A\&A, 315, L309

Dale, D. A., Helou, G., Brauher, J. R., Cutri, R. M., Malhotra, S., \& Beichman, C. A. $2004 \mathrm{a}, \mathrm{ApJ}, 604,565$

Dale, D. A., Helou, G., Contursi, A., Silbermann, N. A., \& Kolhatkar, S. 2001, ApJ, 549, 215

Dale, D. A., et al. 2004b, ApJ, 601, 813 2005, ApJ, 633, 857

Draine, B. T., \& Li, A. 2001, ApJ, 551, 807

Engelbracht, C. W., Gordon, K. D., Rieke, G. H., Werner, M. W., Dale, D. A., \& Latter, W. B. 2005, ApJ, 628, L29

\section{REFERENCES}

Farrah, D., Afonso, J., Efstathiou, A., Rowan-Robinson, M., Fox, M., \& Clements, D. 2003, MNRAS, 343, 585

Förster Schreiber, N. M., Roussel, H., Sauvage, M., \& Charmandaris, V. 2004, A\&A, 419, 501

Galliano, F., Madden, S. C., Jones, A. P., Wilson, C. D., \& Bernard, J.-P. 2005, A\&A, 434, 867

Genzel, R., \& Cesarsky, C. J. 2000, ARA\&A, 38, 761

Genzel, R., et al. 1998, ApJ, 498, 579

Giveon, U., Sternberg, A., Lutz, D., Feuchtgruber, H., \& Pauldrach, A. W. A. 2002, ApJ, 566, 880

González Delgado, R. M., Cid Fernandes, R., Pérez, E., Martins, L. P., StorchiBergmann, T., Schmitt, H., Heckman, T., \& Leitherer, C. 2004, ApJ, 605, 127

Haas, M., Siebenmorgen, R., Schulz, B., Krügel, E., \& Chini, R. 2005, A\&A, 442, L39 
Habing, H. J. 1968, Bull. Astron. Inst. Netherlands, 19, 421

Henry, R. B. C., \& Worthey, G. 1999, PASP, 111, 919

Ho, L., Filippenko, A. V., \& Sargent, W. L. 1993, ApJ, 417, 63 1997a, ApJ, 487, 579 1997b, ApJS, 112, 315

Hollenbach, D., \& Tielens, A. G. G. M. 1999, Rev. Mod. Phys., 71, 173

Houck, J. R., et al. 2004a, ApJS, 154, 18 2004b, ApJS, 154, 211

Kauffmann, G., et al. 2003, MNRAS, 346, 1055

Kaufman, M. J., Wolfire, M. G., \& Hollenbach, D. J. 2006, ApJ, in press

Kennicutt, R. C., Jr., et al. 2003, PASP, 115, 928

Kewley, L. J., Dopita, M. A., Sutherland, R. S., Heisler, C. A., \& Trevena, J. 2001, ApJ, 556, 121

Laurent, O., Mirabel, I. F., Charmandaris, V., Gallais, P., Madden, S. C., Sauvage, M., Vigroux, L., \& Cesarsky, C. 2000, A\&A, 359, 887

Li, A., \& Draine, B. T. 2001, ApJ, 554, 778

Lutz, D., Veilleux, S., \& Genzel, R. 1999, ApJ, 517, L13

Madden, S. C., Galliano, F., Jones, A. P., \& Sauvage, M. 2006, A\&A, 446, 877

Maloney, P. R., Hollenbach, D. J., \& Tielens, A. G. G. M. 1996, ApJ, 466, 561

Martín-Hernández, N. L., Vermeij, R., Tielens, A. G. G. M., van der Hulst, J. M., \& Peeters, E. 2002, A\&A, 389, 286

McCall, M. L. 1982, Ph.D. thesis, Univ. Texas at Austin

Meijerink, R., \& Spaans, M. 2005, A\&A, 436, 397

Moustakas, J., \& Kennicutt, R. C. 2006, ApJS, 164, 81

Osterbrock, D. E. 1993, ApJ, 404, 551

Pagel, B. E. J., \& Edmunds, M. G. 1981, ARA\&A, 19, 77

Peeters, E., Mattioda, A. L., Hudgins, D. M., \& Allamandola, L. J. 2004a, ApJ, 617, L65

Peeters, E., Spoon, H. W. W., \& Tielens, A. G. G. M. 2004b, ApJ, 613, 986

Peeters, E., et al. 2002, A\&A, 381, 571

Pilyugin, L. S., \& Ferrini, F. 1998, A\&A, 336, 103

Rieke, G. H., et al. 2004, ApJS, 154, 25

Roussel, H., Helou, G., Beck, R., Condon, J. J., Bosma, A., Matthews, K., \& Jarrett, T. H. 2003, ApJ, 593, 733
Schaerer, D., \& Stasińska, G. 1999, A\&A, 345, L17

Siebenmorgen, R., Freudling, W., Krügel, E., \& Haas, M. 2004, A\&A, 421, 129

Smith, J. D. T., et al. 2004, ApJS, 154, 199

Spinoglio, L., \& Malkan, M. A. 1992, ApJ, 399, 504

Sturm, E., Lutz, D., Tran, D., Feuchtgruber, H., Genzel, R., Kunze, D., Moorwood, A. F. M., \& Thornley, M. D. 2000, A\&A, 358, 481

Sturm, E., Lutz, D., Verma, A., Netzer, H., Sternberg, A., Moorwood, A. F. M. Oliva, E., \& Genzel, R. 2002, A\&A, 393, 821

Taniguchi, Y., Yoshino, A., Ohyama, Y., \& Nishiura, S. 1999, ApJ, 514, 660

Tayal, S. S., \& Gupta, G. P. 1999, ApJ, 526, 544

Thornley, M. D., Förster Schreiber, N. M., Lutz, D., Genzel, R., Spoon, H. W. W., Kunze, D., \& Sternberg, A. 2000, ApJ, 539, 641

Thuan, T. X., Sauvage, M., \& Madden, S. 1999, ApJ, 516, 783

Tran, Q. D., et al. 2001, ApJ, 552, 527

Veilleux, S. 1991, ApJ, 369, 331

Veilleux, S., \& Osterbrock, D. E. 1987, ApJS, 63, 295

Veilleux, S., Sanders, D. B., \& Kim, D.-C. 1999, ApJ, 522, 139

Verma, A., Lutz, D., Sturm, E., Sternberg, A., Genzel, R., \& Vacca, W. 2003 , A\&A, 403, 829

Vermeij, R., Damour, F., van der Hulst, J. M., \& Baluteau, J.-P. 2002, A\&A, 390, 649

Vermeij, R., \& van der Hulst, J. M. 2002, A\&A, 391, 1081

Verstraete, L., et al. 2001, A\&A, 372, 981

Vila-Costas, M. B., \& Edmunds, M. G. 1992, MNRAS, 259, 121

Voit, G. M. 1992, ApJ, 399, 495

Wang, W., Liu, X.-W., Zhang, Y., \& Barlow, M. J. 2004, A\&A, 427, 873

Weedman, D. W., et al. 2005, ApJ, 633, 706

Wu, Y., Charmandaris, V., Hao, H., Brandl, B. R., Bernard-Salas, J., Spoon, H. W. W., \& Houck, J. R. 2006, ApJ, 639, 157

Xu, C., Lonsdale, C. J., Shupe, D. L., O’Linger, J., \& Masci, F. 2001, ApJ, 562, 179 